\title{
Radiation and Heat Source Effects on MHD Free Convection Flow over an Inclined Porous Plate in the Presence of Viscous Dissipation
}

\author{
Ekakitie Omamoke ${ }^{1, ~ *, ~ E m e k a ~ A m o s ~}{ }^{2}$, Kubugha Wilcox Bunonyo ${ }^{3}$ \\ ${ }^{1}$ Department of Mathematics, Bayelsa Medical University, Yenagoa, Nigeria \\ ${ }^{2}$ Department of Mathematics, Rivers State University, Port Harcourt, Nigeria \\ ${ }^{3}$ Department of Mathematics and Statistics, Federal University Otuoke, Yenagoa, Nigeria \\ Email address: \\ omamoke.ekakitie@bmu.edu.ng (E. Omamoke), ekakitieomamoke@gmail.com (E. Omamoke), amos.emeka@ust.edu.ng (E. Amos), \\ wilcoxbk@fuotuoke.edu.ng (K. W. Bunonyo) \\ ${ }^{*}$ Corresponding author
}

\section{To cite this article:}

Ekakitie Omamoke, Emeka Amos, Kubugha Wilcox Bunonyo. Radiation and Heat Source Effects on MHD Free Convection Flow over an Inclined Porous Plate in the Presence of Viscous Dissipation. American Journal of Applied Mathematics. Vol. 8, No. 4, 2020 , pp. $190-206$. doi: $10.11648 /$ j.ajam.20200804.14

Received: June 18, 2020; Accepted: July 3, 2020; Published: July 17, 2020

\begin{abstract}
This study analyzes radiation, viscous dissipation and heat source effects on magneto-hydrodynamic free convection flow, of a viscous incompressible fluid over an inclined porous plate. Applying the perturbation technique, the solution of a set of ordinary differential equations are gotten as a result of reducing the non-linear partial differential equations of motion, energy and diffusion, which is solved analytically for velocity, temperature and the concentration distribution. The effect of Radiation, viscous dissipation and heat source on the velocity, temperature, concentration, skin friction, heat transfer and rate of mass flux distribution is plotted graphically using Mathematica 12 software and discussed. It is observed that increased Magnetic field reduces the velocity profile, increases the temperature, skin friction and heat transfer profile. Increase in radiation reduces heat transfer causing a mixed effect on the velocity, temperature and skin friction while an increase in the heat source causes a turbulent effect on the velocity, temperature and skin friction profile. Increase in porosity reduces the velocity, temperature, skin friction and heat transfer profile and finally parameters such as Chemical reaction, Grashof concentration number and Schmidt number had no effect on the velocity, temperature, skin friction and heat transfer profile.
\end{abstract}

Keywords: Free Convection, Radiation, Viscous Dissipation, Heat Source, Inclined Plate, Porous Plate, Magneto Hydrodynamic (MHD)

\section{Introduction}

Magneto-hydrodynamic Free convection flow with radiation, viscous dissipation and heat source effect on the boundary layer control and fluid flow affects the performances of engineering, science and medical devices and systems. There are rich application in MHD power generators, nuclear reactors, Human Blood flow and Water flows etc. MHD free convective heat and mass transfer flow for vertical, horizontal and inclined surfaces have attracted many researchers because of its rich application in Medicine, engineering and technology, agriculture, space technology and many more. Amos and Ekakitie [1] studied MHD free convective flow over an inclined porous surface with variable suction and radiation effect while Isreal-Cookey et al. [2] studied the influence of viscous dissipation and radiation on unsteady MHD free-convection flow past an infinite heated vertical plate in a porous medium with time-dependent suction. Mebine [3] studied the thermal solutal MHD flow with radiative heat transfer over oscillating plate in a fluid that is chemically active and got the solutions for the unsteady velocity, temperature and concentration with Laplace transform on the assumption of an optically thin medium, and linear differential approximation model for the radiative flux. Venkateswarlu [4] analyzed the MHD unsteady flow of viscous, incompressible electrical 
conduction fluid over a vertical porous plate under the influence of thermal radiation and chemical reaction while Sankar et al. [5] studied radiation and chemical reaction effects on an unsteady MHD convection flow past a vertical moving porous plate embedded in a porous medium with viscous dissipation. Prasad et al. [6] did a study on the heat and mass transfer for MHD flow of nano fluid with radiation free convective double diffusive and dissipative visco-elastic fluid flow in a porous medium with suction. Venkateswarlu et al. [8] studied Soret, hall current, rotation, chemical reaction and thermal radiation effects on unsteady MHD heat and mass transfer of a natural convection flow past an accelerated vertical plate, Reddy et al. [9] did a study on Soret and Defour effects on radiation absorption fluid in the presence of exponentially varying temperature and concentration in a conducting field and Rojaa et al. [10] studied radiation and chemical reaction effect on MHD free convective flow of a micro polar fluid bounded by a vertical infinite surface with a viscous dissipation and uniform or constant suction. Venkateswarlu et al. [11] did a study on unsteady MHD flow of a viscous fluid past a vertical porous plate under oscillatory suction velocity while Ojemeri et al. [12] studied the effect of Soret and radial magnetic field of a free convective slip flow in a viscous reactive fluid towards a vertical porous cylinder. Reddy et al. [13] studied radiation and mass transfer effects on unsteady MHD free convection flow of an incompressible viscous fluid past a moving vertical cylinder and Rammana Reddy et al. [14] studied the influence of chemical reaction, radiation and rotation on MHD Nano fluid flow past a permeable flat surface in a medium that is porous. Krupa et al. [15] studied the numerical analysis on the effect of diffusion-thermo and thermo-diffusion on two-phase boundary layer flow past a absorption while Rao et al. [7] did a study on Unsteady MHD

stretching sheet with fluid particles suspension and chemical reaction. Salawu and Dada [16] studied radiative heat transfer of variable viscosity and thermal conductivity effects on inclined magnetic field with dissipation in a non-Darcy medium and Das [17] studied the effect of thermal radiation on heat and mass transfer flow of MHD micro-polar fluid in a rotary frame of reference. Ekakitie and Amos [18] studied the impact of chemical reaction and heat source on MHD free convection flow over an inclined porous surface. Hence in this research we will be looking at the effect of radiation, viscous dissipation and heat source on MHD free convective flow over an inclined porous plate. Pertinent parameters such as suction and radiation had an effect on velocity profile, temperature profile, concentration profile and heat transfer.

\section{Mathematical Formulation}

We consider a free convective unsteady flow of a viscous incompressible, chemically reactive, radiative and viscous hydro magnetic fluid over an inclined plate at an angle $\alpha$ in the vertical direction. A magnetic field of constant intensity $\mathrm{B}_{0}$ is applied in the $\mathrm{y}-$ direction. The plate moves uniformly with velocity $\mathrm{V}$ in the $\mathrm{x}$ - direction, the temperature $T_{w}>$ $T_{\infty}$ and concentration $C_{w}>C_{\infty}$ are conditions at the wall and ambient regions respectively. We assume a homogeneous chemical reaction of first and second order and that of Boussinesq approximation is valid. We apply a constant magnetic field of intensity $\mathrm{B}_{0}$ in the $\mathrm{y}$-direction.

Thus the flow equations are as follows.

$$
\frac{\partial \mathrm{v}^{\prime}}{\partial y^{\prime}}=0
$$

$$
\begin{aligned}
& \frac{\partial u^{\prime}}{\partial t^{\prime}}+\mathrm{v}^{\prime} \frac{\partial u^{\prime}}{\partial y^{\prime}}=v \frac{\partial^{2} u^{\prime}}{\partial y^{\prime}}-\left[\frac{\delta_{c} B_{0}^{2}}{\rho}+\frac{v}{k}\right] u^{\prime}+g B_{T}\left(T^{\prime}-T_{\infty}\right) \cos \propto+g B_{C}\left(C^{\prime}-C_{\infty}\right) \cos \propto \\
& \frac{\partial T^{\prime}}{\partial t^{\prime}}+\mathrm{v}^{\prime} \frac{\partial T^{\prime}}{\partial y^{\prime}}=\frac{K}{\rho C_{p}} \frac{\partial^{2} T^{\prime}}{\partial{y^{\prime}}^{2}}+\frac{\mu}{\rho C_{p}}\left[\frac{\partial u^{\prime}}{\partial y^{\prime}}\right]^{2}-\frac{K}{\rho C_{p}} \frac{\partial q_{r}^{\prime}}{\partial y^{\prime}}+\frac{Q_{0}}{\rho C_{p}}\left(T^{\prime}-T_{\infty}\right)+\frac{\delta_{c} B_{0}^{2}}{\rho C_{p}} u^{\prime 2} \\
& \frac{\partial C^{\prime}}{\partial t^{\prime}}+\mathrm{v}^{\prime} \frac{\partial C^{\prime}}{\partial y^{\prime}}=D \frac{\partial^{2} C^{\prime}}{\partial y^{\prime 2}}-K_{r}^{\prime}\left(C^{\prime}-C_{\infty}\right)
\end{aligned}
$$

The velocity $(\mathrm{u})$, temperature $(\mathrm{T})$ and concentration $(\mathrm{C})$ fields in the above equations have the boundary conditions:

$$
\begin{gathered}
u^{\prime}=U ; \mathrm{v}^{\prime}=-\mathrm{V}\left(1+\varepsilon e^{i \omega t}\right) ; T^{\prime}=T_{w}^{\prime} ; C^{\prime}=C_{w}^{\prime} ; \text { at } y=0 \\
u=V(t)=\mathrm{V}^{\prime}\left(1+\varepsilon e^{i \omega t}\right) ; T^{\prime}=T_{\infty}^{\prime} ; C^{\prime}=C_{w}^{\prime} \text { as } y \rightarrow \infty
\end{gathered}
$$

Considering (3) by using the Rosseland diffusion approximation where the radiative heat flux $\mathrm{q}$ is given by

$$
q_{r}^{\prime}=-\frac{4 \delta^{\prime}}{3 k^{\prime}} \frac{\partial T^{\prime}}{\partial y^{\prime}}=-\frac{4 \delta^{\prime}}{3 k^{\prime}} \nabla T^{\prime}
$$

Where $\delta^{\prime}$ is the Stefan - Boltzmann constant and $k^{\prime}$ is the rosseland mean absorption coefficient. Assuming that the temperature differences within the flow are sufficiently small that $T^{\prime}$ may be expressed as a linear function of temperature $T^{\prime}=4 T_{O}^{3} T-3 T_{O}^{\prime}$ This implies that

$$
q_{r}^{\prime}=-\frac{16 \delta^{\prime} T_{O}^{3}}{3 k^{\prime}} \frac{\partial T}{\partial y}
$$


In order to write the governing equations and boundary conditions in dimensionless form, the following non-dimensional quantities are introduced

$$
\begin{gathered}
y=\frac{\mathrm{v} y^{\prime}}{v} ; u=\frac{u^{\prime}}{\mathrm{U}} ; t=\frac{\mathrm{V}^{\prime 2} t^{\prime}}{v} ; \mathrm{v}=\frac{V^{\prime}}{\mathrm{V}} ; \theta=\frac{T^{\prime}-T_{\infty}}{T_{w}^{\prime}-T_{\infty}} ; C=\frac{C^{\prime}-C_{\infty}}{C_{w}^{\prime}-C_{\infty}} \\
M^{2}=\frac{v \delta_{c} B_{O}^{2}}{\rho \mathrm{V}^{2}} ; \mathrm{N}^{2}=\frac{v^{2}}{K \mathrm{~V}^{2}} ; G_{T}=\frac{g v B_{T} \theta\left(T_{w}{ }^{\prime}-T_{\infty}\right)}{\mathrm{UV}^{2}} ; G_{C}=\frac{g v B_{C} C\left(C_{w}{ }^{\prime}-C_{\infty}\right)}{\mathrm{UV}^{2}} ; v=\frac{\mu}{\rho} ; \\
P_{r}=\frac{\mu C_{p}}{K} ; R^{2}=\frac{16 \delta^{\prime} T_{O}^{3}}{K} ; E_{C}=\frac{\mathrm{U}^{2}}{C_{p}\left(T_{w}^{\prime}-T_{\infty}\right)} ; S_{c}=\frac{v}{D} ; H=\frac{Q_{0} v}{\rho C_{p} \mathrm{~V}^{2}}
\end{gathered}
$$

The momentum, energy and diffusion equation in dimensionless form is written as

$$
\begin{gathered}
\frac{\partial u}{\partial t}-\left(1+\varepsilon e^{i \omega t}\right) \frac{\partial u}{\partial y}=\frac{\partial^{2} u}{\partial y^{2}}-\left[\left(M^{2}+N^{2}\right) u\right]+G_{T} \theta \cos \propto+G_{C} C \cos \propto \\
P_{r} \frac{\partial \theta}{\partial t}-P_{r}\left(1+\varepsilon e^{i \omega t}\right) \frac{\partial \theta}{\partial y}=\left(1+R^{2}\right) \frac{\partial^{2} \theta}{\partial y^{2}}+P_{r} E_{C}\left(\frac{\partial u}{\partial y}\right)^{2}+H \operatorname{Pr} \theta+M^{2} \operatorname{Pr} E c u
\end{gathered}
$$

$$
S_{c} \frac{\partial C}{\partial t}-S_{c}\left(1+\varepsilon e^{i \omega t}\right) \frac{\partial C}{\partial y}=\frac{\partial^{2} C}{\partial y^{2}}-S_{c} K_{r} C
$$

The corresponding boundary conditions in nondimensional form are:

$$
\begin{gathered}
u=\mathrm{U}=0, \theta=1, C=1, \text { at } y=0 \\
u \rightarrow 1+\varepsilon e^{i \omega t}, \theta \rightarrow 0, C \rightarrow 0, \text { at } y \rightarrow \infty
\end{gathered}
$$

In the above equations $\mathrm{u}$ and $\mathrm{v}$ are the velocity components, $\mathrm{x}$ and $\mathrm{y}$ are the Cartesian coordinates, $\alpha$ is the angle of inclination in the vertical direction of the semi-infinite moving plate. $t$ is the time, $T_{w}^{\prime}$ is the temperature at the wall, $T_{\infty}^{\prime}$ is the reference temperature and $g$ is the acceleration due to gravity. $\mathrm{V}$ is the suction velocity, $B_{0}$ is the constant magnetic field, $\mathrm{k}$ is the porosity parameter, $C_{p}$ is the specific heat capacity and $M$ is the magnetic parameter. $G_{T}$ is Grashof temperature number, $P_{r}$ is the Prandtl number, Sc is Schmidt number, $\mathrm{R}$ is the radiation parameter, $q_{r}^{\prime}$ is the radiation heat flux, Ec is the Eckert number, $\varepsilon$ is the small positive constant, $\rho$ is the density, $\beta_{T}$ is the coefficient of volume expansion due to temperature and $\beta_{C}$ is the coefficient of volume expansion due to concentration. $N$ is the porosity number, $\omega$ is the free stream frequency oscillation, $\partial_{c}$ is the electrical conductivity, $G_{c}$ is the Grashof diffusion number, $\mathrm{Kr}$ is the chemical reaction parameter, $\theta$ is the temperature and $C_{w}$ is the concentration at the wall.

\section{Method of Solution}

Equations (2) - (4) are coupled nonlinear partial differential equations that is solved analytically and then reduced to a set of ordinary differential equations with the expressions for velocity $(\mathrm{u})$, temperature $(\theta)$ and concentration (C) of the fluid in dimensionless form as follows:

$$
\begin{aligned}
& u(y, t)=u_{o}(y)+\varepsilon e^{i \omega t} u_{1}+0\left(\varepsilon^{2}\right) \\
& \theta(y, t)=\theta_{o}(y)+\varepsilon e^{i \omega t} \theta_{1}+0\left(\varepsilon^{2}\right) \\
& C(y, t)=C_{o}(y)+\varepsilon e^{i \omega t} C_{1}+0\left(\varepsilon^{2}\right)
\end{aligned}
$$

Substituting equation (15) to (17) in the set of equations (11), (12) and (13) and equating non - harmonic and harmonic terms and neglecting the higher order terms of $0\left(\varepsilon^{2}\right)$, the following set of ordinary differential equations are obtained with their boundary conditions.

$$
\begin{gathered}
u_{0}+u_{0}-\left(\mathrm{N}^{2}+M^{2}\right) u_{0}=-G_{T} \theta_{0} \cos \propto-G_{C} C_{0} \cos \propto \\
\left(1+R^{2}\right) \theta_{0}+P_{r} \theta_{0}+H \operatorname{Pr} \theta_{0}=-P_{r} E_{c} u_{0}{ }^{2}-M^{2} \operatorname{PrEc} u_{0} \\
C_{0}+S_{c} C_{0}-S_{c} K_{r} C_{o}=0 \\
u_{1}+u_{1}-\left(\mathrm{N}^{2}+M^{2}+i \omega\right) u_{1}=-u_{0}-G_{T} \theta_{1} \cos \propto-G_{C} C_{1} \cos \propto \\
\left(1+R^{2}\right) \theta_{1}+P_{r} \theta_{1}+\left[H P r-P_{r} i \omega\right] \theta_{1}=-P_{r} \theta_{0}-2 P_{r} E_{c} u_{0} u_{1}-M^{2} \operatorname{PrEcu} u_{1} \\
C_{1}+S_{c} C_{1}-\left(S_{c} K_{r}+S_{c} i \omega\right) C_{1}=-S_{c} C_{0}
\end{gathered}
$$

Boundary conditions

$$
\begin{aligned}
& u_{0}=u ; u_{1}=0 ; \theta_{0}=1 ;, \theta_{1}=0 ; C_{0}=1 ; C_{1}=0 \text { at } y=0 \\
& u_{0} \rightarrow 0 ; u_{1} \rightarrow 0 ; \theta_{0} \rightarrow 0 ;, \theta_{1} \rightarrow 0 ; C_{0} \rightarrow 0 ; C_{1} \rightarrow 0 \text { as } y \rightarrow \infty
\end{aligned}
$$

For $0(\varepsilon)$ equations

To solve the nonlinear coupled equations (18) - (23) we assume that the dissipation parameter (Eckert number $\left.E_{c} \ll 1\right)$ is 
small, and therefore, advance an asymptotic expansion for the flow temperature and velocity as follows using the boundary condition ins equation $(24-25)$ :

$$
\begin{aligned}
& u_{0}(y)=u_{o 1}(y)+E_{c} u_{o 2}(y) \\
& \theta_{0}(y)=\theta_{o 1}(y)+E_{c} \theta_{o 2}(y) \\
& u_{1}(y)=u_{11}(y)+E_{c} u_{12}(y) \\
& \theta_{1}(y)=\theta_{11}(y)+E_{c} \theta_{12}(y)
\end{aligned}
$$

Substituting Eq. (26) into Eq. (18) - (23) we obtain the following sequence of approximation

$$
\begin{gathered}
u_{01}+u_{01}-\left(\mathrm{N}^{2}+M^{2}\right) u_{01}-G_{T} \theta_{01} \cos \propto-G_{C} C_{0} \cos \propto \\
\left(1+R^{2}\right) \theta_{01}+P_{r} \theta_{01}+\operatorname{HPr} \theta_{01}=0 \\
u_{02}+u_{02}-\left(M^{2}+\mathrm{N}^{2}\right) u_{o 2}=-G_{T} \theta_{02} \cos \propto \\
\left(1+R^{2}\right) \theta_{02}+P_{r} \theta_{02}+H \operatorname{Pr} \theta_{02}=-P_{r} u_{01}{ }^{2}-M^{2} P r u_{01}
\end{gathered}
$$

Boundary conditions

$$
\begin{gathered}
u_{01}=u ; \theta_{01}=1 ; u_{02}=0=\theta_{02} \text { at } y=0 \\
u_{01} \rightarrow 0 ; \theta_{01} \rightarrow 0 ; u_{02} \rightarrow 0 ; \theta_{02} \rightarrow 0 ; \text { as } y \rightarrow \infty \\
u_{11}+u_{11}-\left(\mathrm{N}^{2}+M^{2}+i \omega\right) u_{11}=-u_{01}-G_{T} \theta_{11} \cos \propto-G_{C} C_{1} \cos \propto \\
\left(1+R^{2}\right) \theta_{11}+\operatorname{Pr} \theta_{11}+[\operatorname{HPr}-\operatorname{Pri\omega }] \theta_{11}=-\operatorname{Pr} \theta_{01} \\
u_{12}+u_{12}-\left(\mathrm{N}^{2}+M^{2}+i \omega\right) u_{12}=-u_{02}-G_{T} \theta_{12} \cos \propto \\
\left(1+R^{2}\right) \theta_{12}+\operatorname{Pr} \theta_{12}+[\operatorname{HPr}-\operatorname{Pri\omega }] \theta_{12}=-\operatorname{Pr} \theta_{02}-2 \operatorname{Pru}_{11} u_{01}-M^{2} \operatorname{Pru}_{11}
\end{gathered}
$$

Boundary condition

$$
\begin{gathered}
u_{11}=0 ; \theta_{11}=0 ; u_{12}=0=\theta_{12} ; \text { at } y=0 \\
u_{11} \rightarrow 0 ; \theta_{11} \rightarrow 0 ; u_{12} \rightarrow 0 ; \theta_{12} \rightarrow 0 ; \text { as } y \rightarrow \infty
\end{gathered}
$$

For $\mathrm{O}(\mathrm{Ec})$ equations solving equations (27) - (30) with the boundary conditions (30) - (31) and equation (32) - (35) satisfying the boundary condition (37) - (38) then equation (25) becomes

$$
\begin{aligned}
& u_{0}(y)=A_{1} e^{-m_{3} y}-A_{2} e^{-m_{2} y}-A_{3} e^{-m_{1} y}-1+E_{c}\left[A_{14} e^{-m_{5} y}-A_{15} e^{-m_{4} y}+A_{16} e^{-2 m_{3} y}+A_{17} e^{-2 m_{2} y}+A_{18} e^{-2 m_{1} y}-\right. \\
& \left.A_{19} e^{-\left(m_{2}+m_{3}\right) y}-A_{20} e^{-\left(m_{1}+m_{3}\right) y}+A_{21} e^{-\left(m_{1}+m_{2}\right) y}+A_{22} e^{-m_{3} y}-A_{23} e^{-m_{2} y}+A_{24} e^{-m_{1} y}\right] \\
& \begin{array}{c}
\theta_{0}(y)=e^{-m_{2} y}+E_{c}\left[A_{4} e^{-m_{4} y}-A_{5} e^{-2 m_{3} y}-A_{6} e^{-2 m_{2} y}-A_{7} e^{-2 m_{1} y}+A_{8} e^{-\left(m_{2}+m_{3}\right) y}+A_{9} e^{-\left(m_{1}+m_{3}\right) y}-A_{10} e^{-\left(m_{1}+m_{2}\right) y}-\right. \\
\left.A_{11} e^{-m_{3} y}+A_{12} e^{-m_{2} y}+A_{13} e^{-m_{1} y}\right]
\end{array} \\
& \begin{array}{c}
\theta_{0}(y)=e^{-m_{2} y}+E_{c}\left[A_{4} e^{-m_{4} y}-A_{5} e^{-2 m_{3} y}-A_{6} e^{-2 m_{2} y}-A_{7} e^{-2 m_{1} y}+A_{8} e^{-\left(m_{2}+m_{3}\right) y}+A_{9} e^{-\left(m_{1}+m_{3}\right) y}-A_{10} e^{-\left(m_{1}+m_{2}\right) y}-\right. \\
\left.A_{11} e^{-m_{3} y}+A_{12} e^{-m_{2} y}+A_{13} e^{-m_{1} y}\right]
\end{array} \\
& u_{1}(y)=A_{29} e^{-m_{8} y}-A_{30} e^{-m_{7} y}-A_{32} e^{-m_{6} y}-A_{34} e^{-m_{3} y}-\left(A_{31}+A_{35}\right) e^{-m_{2} y}-\left(A_{33}+A_{36}\right) e^{-m_{1} y}+E_{c}\left[A_{60} e^{-m_{10} y}-\right. \\
& A_{61} e^{-m_{9} y}-A_{62} e^{-m_{4} y}+A_{63} e^{-m_{3} y}-A_{64} e^{-m_{2} y}-A_{65} e^{-m_{1} y}+A_{60} e^{-2 m_{3} y}+A_{61} e^{-2 m_{2} y}+A_{62} e^{-2 m_{1} y}-A_{63} e^{-\left(m_{2}+m_{3}\right) y}- \\
& A_{64} e^{-\left(m_{1}+m_{3}\right) y}-A_{65} e^{-\left(m_{1}+m_{2}\right) y}+A_{66} e^{-m_{8} y}-A_{67} e^{-m_{7} y}-A_{68} e^{-m_{6} y}-A_{69} e^{-m_{5} y}+A_{70} e^{-2 m_{3} y}+A_{71} e^{-2 m_{2} y}+A_{72} e^{-2 m_{1} y}- \\
& A_{73} e^{-\left(m_{2}+m_{3}\right) y}-A_{74} e^{-\left(m_{1}+m_{3}\right) y}+A_{75} e^{-\left(m_{1}+m_{2}\right) y}+A_{76} e^{-\left(m_{3}+m_{8}\right) y}--A_{77} e^{-\left(m_{3}+m_{7}\right) y-}-A_{78} e^{-\left(m_{3}+m_{6}\right) y}+A_{79} e^{-\left(m_{2}+m_{8}\right) y}+ \\
& \left.A_{80} e^{-\left(m_{2}+m_{7}\right) y}+A_{81} e^{-\left(m_{2}+m_{6}\right) y}-A_{82} e^{-\left(m_{1}+m_{8}\right) y}+A_{83} e^{-\left(m_{1}+m_{7}\right) y}+A_{84} e^{-\left(m_{1}+m_{6}\right) y}\right] \\
& \theta_{1}(y)=A_{27} e^{-m_{7} y}+A_{28} e^{-m_{2} y}+E_{c}\left[A_{37} e^{-m_{9} y}+A_{38} e^{-m_{4} y}-A_{39} e^{-2 m_{3} y}-A_{40} e^{-2 m_{2} y}-A_{41} e^{-2 m_{1} y}+A_{42} e^{-\left(m_{2}+m_{3}\right) y}+\right. \\
& A_{43} e^{-\left(m_{1}+m_{3}\right) y}-A_{44} e^{-\left(m_{1}+m_{2}\right) y}-A_{45} e^{-m_{3} y}+A_{46} e^{-m_{2} y}+A_{47} e^{-m_{1} y}-A_{48} e^{-\left(m_{3}+m_{8}\right) y}+A_{49} e^{-\left(m_{3}+m_{7}\right) y}+ \\
& A_{50} e^{-\left(m_{3}+m_{6}\right) y}-A_{51} e^{-\left(m_{2}+m_{8}\right) y}-A_{52} e^{-\left(m_{2}+m_{7}\right) y}-A_{53} e^{-\left(m_{2}+m_{6}\right) y}+A_{54} e^{-\left(m_{1}+m_{8}\right) y}-A_{55} e^{-\left(m_{1}+m_{7}\right) y}- \\
& \left.A_{56} e^{-\left(m_{1}+m_{6}\right) y}-A_{57} e^{-m_{8} y}+A_{58} e^{-m_{7} y}+A_{59} e^{-m_{6} y}\right]
\end{aligned}
$$

Substituting equation (39) - (42) into equation (15) - (16) we obtain the velocity, temperature and concentration profiles respectively as

$$
u(y, t)=A_{1} e^{-m_{3} y}-A_{2} e^{-m_{2} y}-A_{3} e^{-m_{1} y}+E_{c}\left[A_{14} e^{-m_{5} y}-A_{15} e^{-m_{4} y}+A_{16} e^{-2 m_{3} y}+A_{17} e^{-2 m_{2} y}+A_{18} e^{-2 m_{1} y}-\right.
$$




$$
\begin{aligned}
& \left.A_{19} e^{-\left(m_{2}+m_{3}\right) y}-A_{20} e^{-\left(m_{1}+m_{3}\right) y}+A_{21} e^{-\left(m_{1}+m_{2}\right) y}+A_{22} e^{-m_{3} y}-A_{23} e^{-m_{2} y}+A_{24} e^{-m_{1} y}\right]+\varepsilon e^{i \omega t}\left\{A_{29} e^{-m_{8} y}-\right. \\
& A_{30} e^{-m_{7} y}-A_{32} e^{-m_{6} y}-A_{34} e^{-m_{3} y}-\left(A_{31}+A_{35}\right) e^{-m_{2} y}-\left(A_{33}+A_{36}\right) e^{-m_{1} y}+E_{c}\left[A_{60} e^{-m_{10} y}-A_{61} e^{-m_{9} y}-A_{62} e^{-m_{4} y}+\right. \\
& A_{63} e^{-m_{3} y}-A_{64} e^{-m_{2} y}-A_{65} e^{-m_{1} y}+A_{60} e^{-2 m_{3} y}+A_{61} e^{-2 m_{2} y}+A_{62} e^{-2 m_{1} y}-A_{63} e^{-\left(m_{2}+m_{3}\right) y}-A_{64} e^{-\left(m_{1}+m_{3}\right) y}- \\
& A_{65} e^{-\left(m_{1}+m_{2}\right) y}+A_{66} e^{-m_{8} y}-A_{67} e^{-m_{7} y}-A_{68} e^{-m_{6} y}-A_{69} e^{-m_{5} y}+A_{70} e^{-2 m_{3} y}+A_{71} e^{-2 m_{2} y}+A_{72} e^{-2 m_{1} y}- \\
& A_{73} e^{-\left(m_{2}+m_{3}\right) y}-A_{74} e^{-\left(m_{1}+m_{3}\right) y}+A_{75} e^{-\left(m_{1}+m_{2}\right) y}+A_{76} e^{-\left(m_{3}+m_{8}\right) y}--A_{77} e^{-\left(m_{3}+m_{7}\right) y-}-A_{78} e^{-\left(m_{3}+m_{6}\right) y}+ \\
& \left.\left.A_{79} e^{-\left(m_{2}+m_{8}\right) y}+A_{80} e^{-\left(m_{2}+m_{7}\right) y}+A_{81} e^{-\left(m_{2}+m_{6}\right) y}-A_{82} e^{-\left(m_{1}+m_{8}\right) y}+A_{83} e^{-\left(m_{1}+m_{7}\right) y}+A_{84} e^{-\left(m_{1}+m_{6}\right) y}\right]\right\} \\
& \theta(y, t)= \\
& e^{-m_{2} y}+E_{c}\left[A_{4} e^{-m_{4} y}-A_{5} e^{-2 m_{3} y}-A_{6} e^{-2 m_{2} y}-A_{7} e^{-2 m_{1} y}+A_{8} e^{-\left(m_{2}+m_{3}\right) y}+A_{9} e^{-\left(m_{1}+m_{3}\right) y}-A_{10} e^{-\left(m_{1}+m_{2}\right) y}-\right. \\
& \left.A_{11} e^{-m_{3} y}+A_{12} e^{-m_{2} y}+A_{13} e^{-m_{1} y}\right]+\varepsilon e^{i \omega t}\left\{A_{27} e^{-m_{7} y}+A_{28} e^{-m_{2} y}+E_{c}\left[A_{37} e^{-m_{9} y}+A_{38} e^{-m_{4} y}-A_{39} e^{-2 m_{3} y}-\right.\right. \\
& A_{40} e^{-2 m_{2} y}-A_{41} e^{-2 m_{1} y}+A_{42} e^{-\left(m_{2}+m_{3}\right) y}+A_{43} e^{-\left(m_{1}+m_{3}\right) y}-A_{44} e^{-\left(m_{1}+m_{2}\right) y}-A_{45} e^{-m_{3} y}+A_{46} e^{-m_{2} y}+A_{47} e^{-m_{1} y}- \\
& A_{48} e^{-\left(m_{3}+m_{8}\right) y}+A_{49} e^{-\left(m_{3}+m_{7}\right) y}+A_{50} e^{-\left(m_{3}+m_{6}\right) y}-A_{51} e^{-\left(m_{2}+m_{8}\right) y}-A_{52} e^{-\left(m_{2}+m_{7}\right) y}-A_{53} e^{-\left(m_{2}+m_{6}\right) y}+ \\
& \left.\left.A_{54} e^{-\left(m_{1}+m_{8}\right) y}-A_{55} e^{-\left(m_{1}+m_{7}\right) y}-A_{56} e^{-\left(m_{1}+m_{6}\right) y}-A_{57} e^{-m_{8} y}+A_{58} e^{-m_{7} y}+A_{59} e^{-m_{6} y}\right]\right\} \\
& C(y, t)=e^{-m_{1} y}+\varepsilon e^{i \omega t}\left\{A_{25} e^{-m_{6} y}+A_{26} e^{-m_{1} y}\right\}
\end{aligned}
$$

The physical quantities of interest are the wall shear stress $\tau_{w}$ is given by

$$
\tau_{w}=\mu \frac{\partial u^{I}}{\partial y^{I}}=\rho v_{0}^{2} u^{I}(0)
$$

The local skin friction factor

$$
\begin{gathered}
C_{f x}=\frac{\tau_{w}}{\rho v_{0}^{2}}=u^{I}(0)=-m_{3} A_{1}+m_{2} A_{2}+m_{1} A_{3}+E_{c}\left[-m_{5} A_{14}+m_{4} A_{15}-2 m_{3} A_{16}-2 m_{2} A_{17}-2 m_{1} A_{18}+\left(m_{2}+m_{3}\right) A_{19}+\right. \\
\left.\left(m_{1}+m_{3}\right) A_{20}-\left(m_{1}+m_{2}\right) A_{21}-m_{3} A_{22}+m_{2} A_{23}+m_{1} A_{24}\right]+\varepsilon\left[-m_{8} A_{29}+m_{7} A_{30}+m_{2}\left(A_{31}+A_{35}\right)+m_{6} A_{32}+\right. \\
m_{1} A_{33}-m_{3} A_{34}+m_{2} A_{35}+m_{1} A_{36}+E_{c}\left(-m_{10} A_{60}+m_{9} A_{61}+m_{4} A_{62}-m_{3} A_{63}+m_{2} A_{64}+m_{1} A_{65}-m_{8} A_{66}+m_{7} A_{67}+\right. \\
m_{6} A_{68}+m_{5} A_{69}-2 m_{3} A_{70}-2 m_{2} A_{71}-2 m_{1} A_{72}+\left(m_{2}+m_{3}\right) A_{73}+\left(m_{1}+m_{3}\right) A_{74}-\left(m_{1}+m_{2}\right) A_{75}-\left(m_{3}+m_{8}\right) A_{76}+ \\
\left(m_{3}+m_{7}\right) A_{77}+\left(m_{3}+m_{6}\right) A_{78}-\left(m_{2}+m_{8}\right) A_{79}-\left(m_{2}+m_{7}\right) A_{80}-\left(m_{2}+m_{6}\right) A_{81}+\left(m_{1}+m_{8}\right) A_{82}-\left(m_{1}+m_{7}\right) A_{83}- \\
\left.\left(m_{1}+m_{6}\right) A_{84}\right]
\end{gathered}
$$

The Local Surface heat Flux $q_{r}=\frac{-4 \delta^{I} \partial T^{I}}{3 k \partial y^{I}}$ where $k$ is the effective thermal conductivity

Local Nusselt Number $N_{u x}=\frac{q_{r}}{T_{w}-T_{\infty}}$ where $q_{r}=\frac{-16 \delta^{I} T_{0}^{3} V_{0}^{I}\left(T_{w}-T_{\infty}\right)}{3 k^{I} v} \frac{\partial \theta}{\partial y}$

Then $N_{u x}=\frac{-16 \delta^{I} T_{0}^{3} V_{0}^{I}}{3 k^{I} v} \frac{\partial \theta}{\partial y}$ where $R_{e x}=\frac{-16 \delta^{I} T_{0}^{3} v_{0}^{I}}{3 k^{I} v}$

$$
\begin{array}{r}
\frac{N_{u x}}{R_{e x}}=\frac{\partial \theta}{\partial y}=\theta^{I}(0)=-m_{2}+E_{c}\left[-m_{4} A_{4}+2 m_{3} A_{5}+2 m_{2} A_{6}+2 m_{1} A_{7}-\left(m_{2}+m_{3}\right) A_{8}=-\left(m_{1}+m_{3}\right) A_{9}+\left(m_{1}+\right.\right. \\
\left.\left.m_{2}\right) A_{10}+m_{3} A_{11}-m_{2} A_{12}-m_{1} A_{13}\right]+\varepsilon\left[-m_{7} A_{27}-m_{2} A_{28}+E_{c}\left(-m_{9} A_{37}-m_{4} A_{38}+2 m_{3} A_{39}+2 m_{2} A_{40}+2 m_{1} A_{41}-\right.\right. \\
\left(m_{2}+m_{3}\right) A_{42}-\left(m_{1}+m_{3}\right) A_{43}+\left(m_{1}+m_{2}\right) A_{44}+m_{3} A_{45}-m_{2} A_{46}-m_{1} A_{47}+\left(m_{3}+m_{8}\right) A_{48}-\left(m_{3}+m_{7}\right) A_{49}- \\
\left(m_{3}+m_{6}\right) A_{50}+\left(m_{2}+m_{8}\right) A_{51}+\left(m_{2}+m_{7}\right) A_{52}+\left(m_{2}+m_{6}\right) A_{53}-\left(m_{1}+m_{8}\right) A_{54}+\left(m_{1}+m_{7}\right) A_{55}+\left(m_{1}+m_{6}\right) A_{56}+ \\
\left.\left.m_{8} A_{57}-m_{7} A_{58}-m_{6} A_{59}\right)\right]
\end{array}
$$

The local surface mass flux is given by

\section{Results and Discussions}

The effect of various parameters on the velocity, temperature, concentration, skin friction, heat transfer and mass flux rate is discussed in this session. An increase in magnetic field reduced the velocity profile in Figure 1 and then increased the temperature profile in Figure 11. This increases the skin friction and increases the heat transfer in Figure 20 and Figure 25. The reduction of the velocity flow was due to Lorentz force which thickens the boundary layer and causes increase in skin friction and decrease in the heat transfer.

$$
\frac{S h_{x}}{R_{e x}}=-\frac{\partial C}{\partial y}=C^{I}(0)=-m_{1}-\varepsilon\left(m_{6} A_{25}+m_{1} A_{26}\right)
$$

An increase in radiation causes a decrease in the velocity and temperature profile in Figure 2 and Figure 9. This in turn reduces both the skin friction in Figure 19 and the heat transfer profile in Figure 24.

An increase in heat source had a mixed effect on the velocity profile in Figure 3, temperature profile decreases near the plate in Figure 10 and skin friction profile reduces in Figure 18 which decreases the heat transfer profile in Figure 23.

In Figure 4, Figure 12, Figure 21 and Figure 26, an increase in porosity reduces the velocity, temperature, skin friction and heat transfer profile. There was an opposite 
effect in Figure 6, Figure 15, Figure 22 and Figure 27 where an increase in viscous dissipation increases the velocity, temperature, skin friction and heat transfer profile.

In Figure 5 and Figure 13, an increase in Grashof number increases the velocity and temperature profile while an increase in Prandtl number in Figure 8 increases the velocity profile. In Figure 7 and Figure 14, an increase in the angle of inclination causes a reduction in velocity and temperature profile near the plate but an increase in velocity and temperature profile at the boundary layer.

In Figure 16 an increase in chemical reaction reduces the concentration profile while an increase in Schmidt number reduces the concentration profile in Figure 17, but increases the mass transfer in figure 28. Figures 29, 30 and 31 shows that there was no effect of Chemical reaction $(\mathrm{Kr})$, Schmidt number (Sc) and Grashof diffusion number (Gc) on the velocity profile.

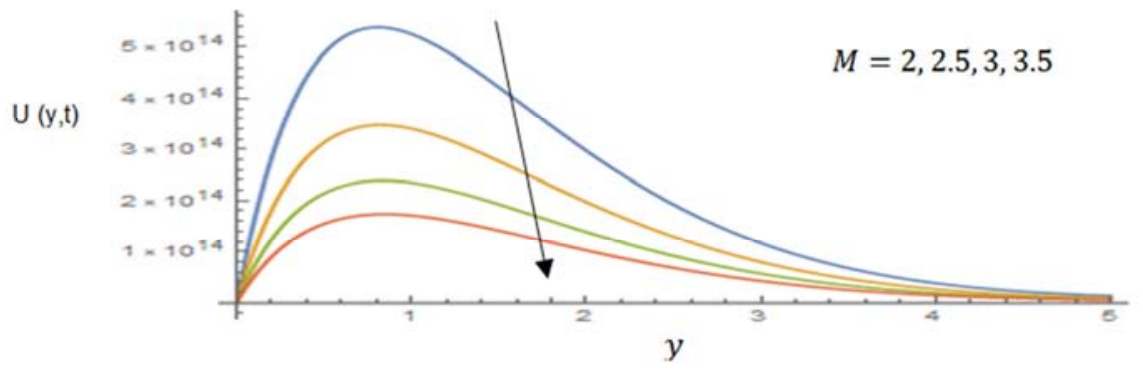

Figure 1. Velocity Profile with Variation of Magnetic Field Parameter $M$ for $H=0.5, P_{r}=0.71, G_{r}=15, G_{c}=10, S_{c}=0.22, K r=2, E c=0.01, R=$ $0.1, N=0.1, \alpha=10, \omega=1, t=1, \varepsilon=1$.
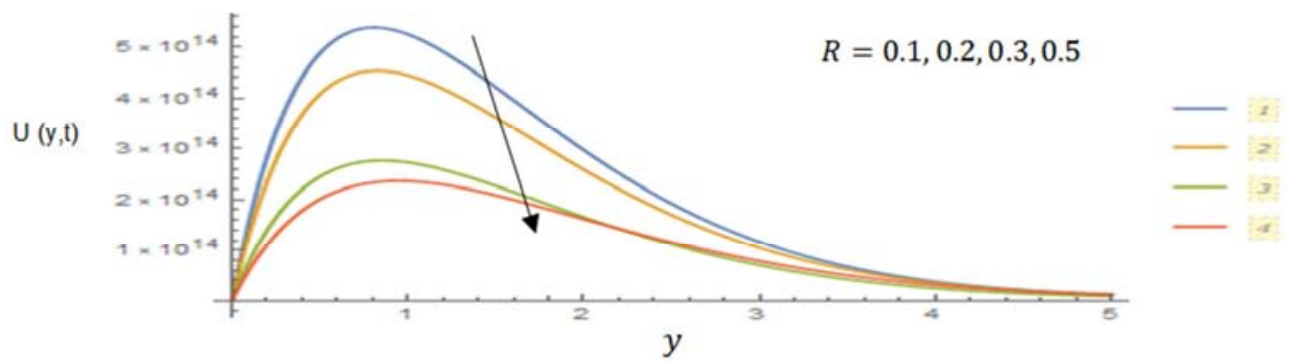

Figure 2. Velocity Profile with Variation of Radiation Parameter $R$ for $M=2, H=0.5, P_{r}=0.71, G_{r}=15, G_{c}=10, S_{c}=0.22, K r=2, E c=0.01, N=$ $0.1, \alpha=10, \omega=1, t=1, \varepsilon=1$.

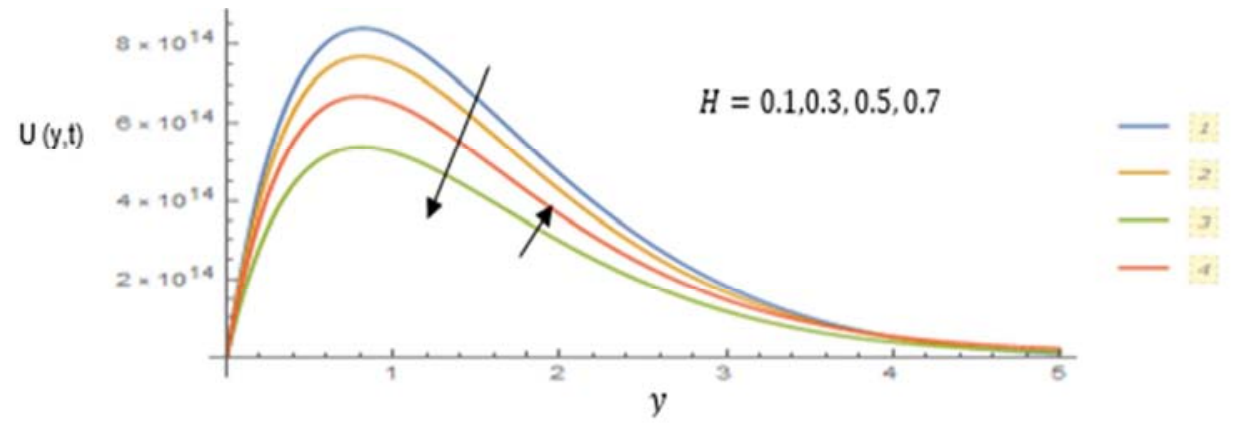

Figure 3. Velocity Profile with Variation of Heat Source Parameter for $H$ for $M=2, P_{r}=0.71, G_{r}=15, G_{c}=10, S_{c}=0.22, K r=2, E c=0.01, R=$ $0.1, N=0.1, \alpha=10, \omega=1, t=1, \varepsilon=1$.

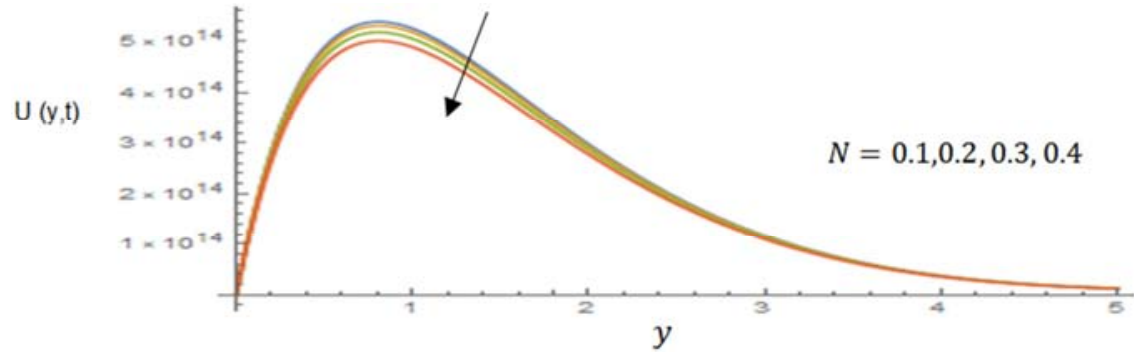

Figure 4. Velocity Profile with Variation of Porosity Parameter $N$ for $M=2, H=0.5, P_{r}=0.71, G_{r}=15, G_{c}=10, S_{c}=0.22, K r=2, E c=0.01, R=$ $0.1, \alpha=10, \omega=1, t=1, \varepsilon=1$. 


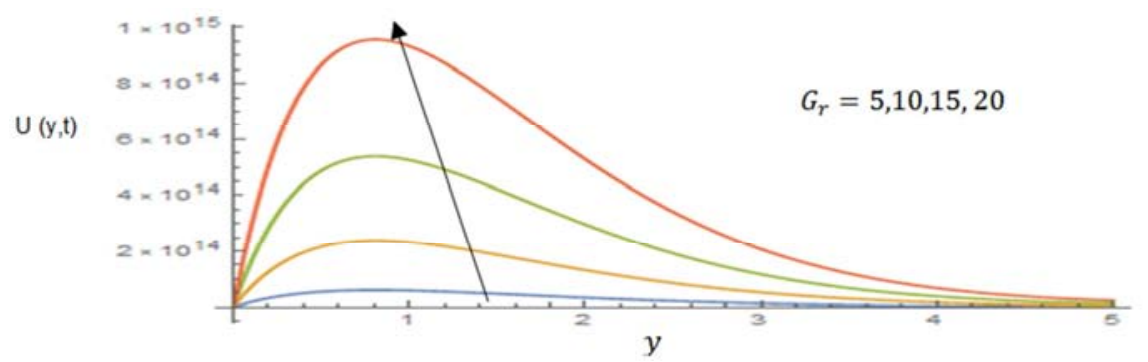

Figure 5. Velocity Profile with Variation of Grashof Temperature Parameter $G_{r}$ for $M=2, H=0.5, P_{r}=0.71, G_{c}=10, S_{c}=0.22, K r=2, E c=0.01, R=$ $0.1, N=0.1, \alpha=10, \omega=1, t=1, \varepsilon=1$.

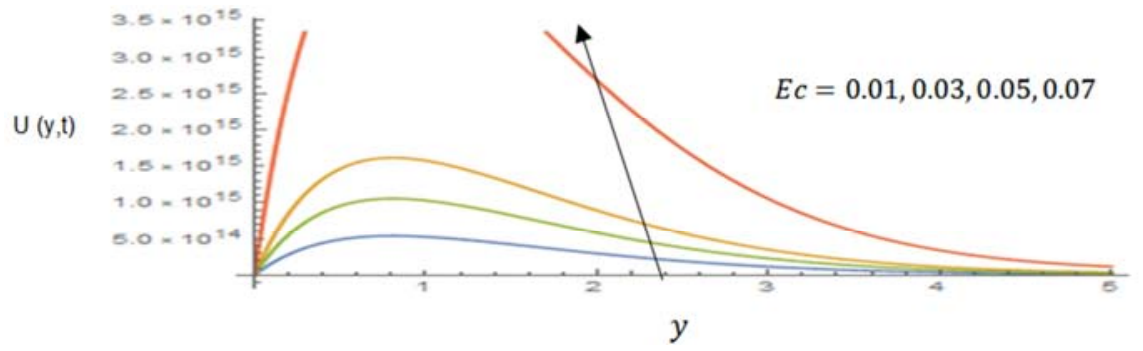

Figure 6. Velocity Profile with Variation of Viscous Dissipation Parameter Ec for $M=2, H=0.5, P_{r}=0.71, G_{r}=15, G_{c}=10, S_{c}=0.22, K r=2, R=$ $0.1, N=0.1, \alpha=10, \omega=1, t=1, \varepsilon=1$.

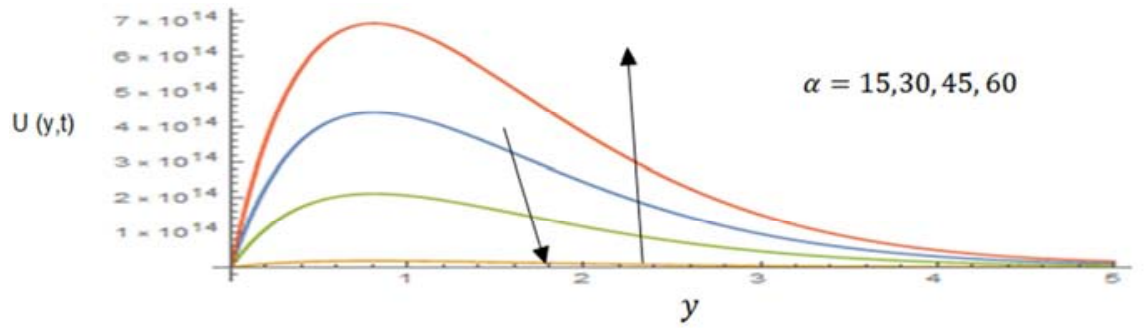

Figure 7. Velocity Profile with Variation of angle of inclination Parameter $\alpha$ for $M=2, H=0.5, P_{r}=0.71, G_{r}=15, G_{c}=10, S_{c}=0.22, K r=2, E c=$ $0.01, R=0.1, N=0.1, \omega=1, t=1, \varepsilon=1$.

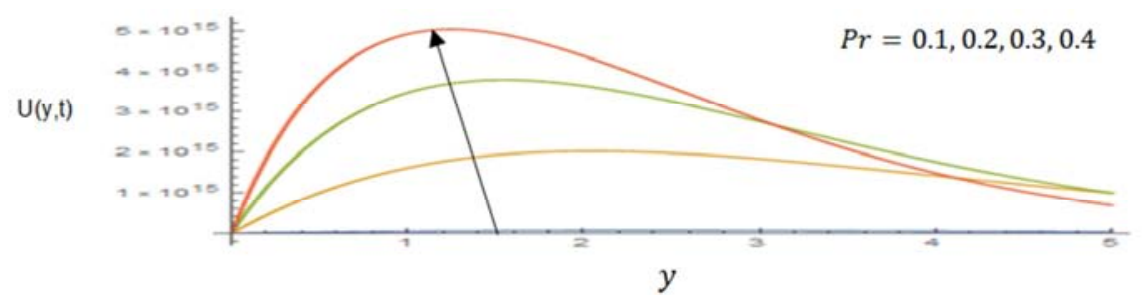

Figure 8. Velocity Profile with Variation of Prandtl Number Parameter Pr for $M=2, H=0.5, G_{r}=15, G_{c}=10, S_{c}=0.22, K r=2, E c=0.01, R=0.1, N=$ $0.1, \alpha=10, \omega=1, t=1, \varepsilon=1$.

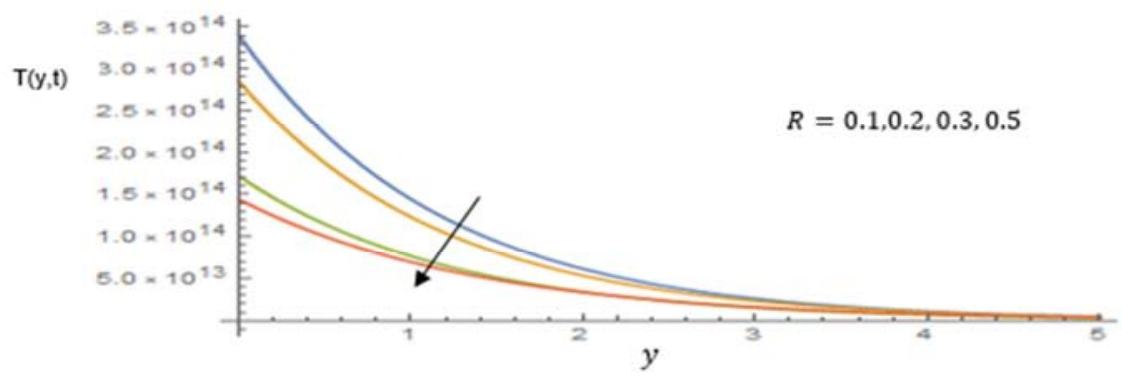

Figure 9. Temperature Profile with Variation of Radiation Parameter $R$ for $M=2, H=0.5, P_{r}=0.71, G_{r}=15, G_{c}=10, S_{c}=0.22, K r=2, E c=0.01, N=$ $0.1, \alpha=10, \omega=1, t=1, \varepsilon=1$. 


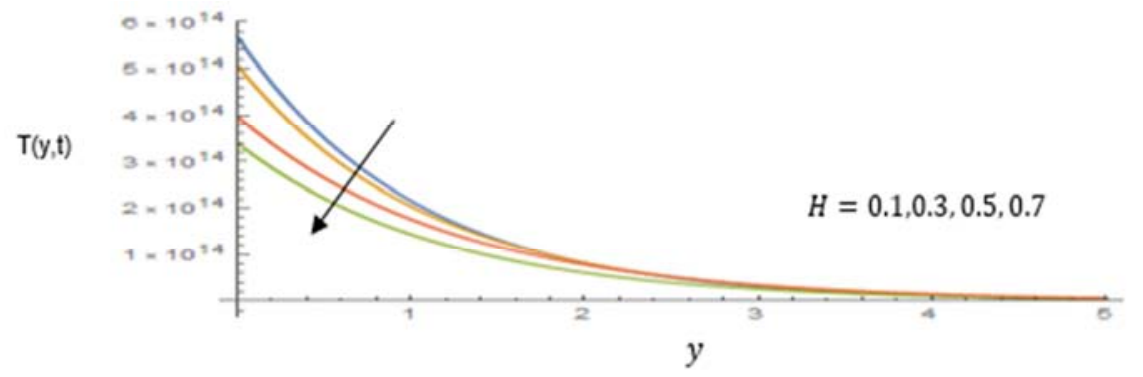

Figure 10. Temperature Profile with Variation of Heat Source Parameter $H$ for $M=2, P_{r}=0.71, G_{r}=15, G_{c}=10, S_{c}=0.22, K r=2, E c=0.01, R=$ $0.1, N=0.1, \alpha=10, \omega=1, t=1, \varepsilon=1$.

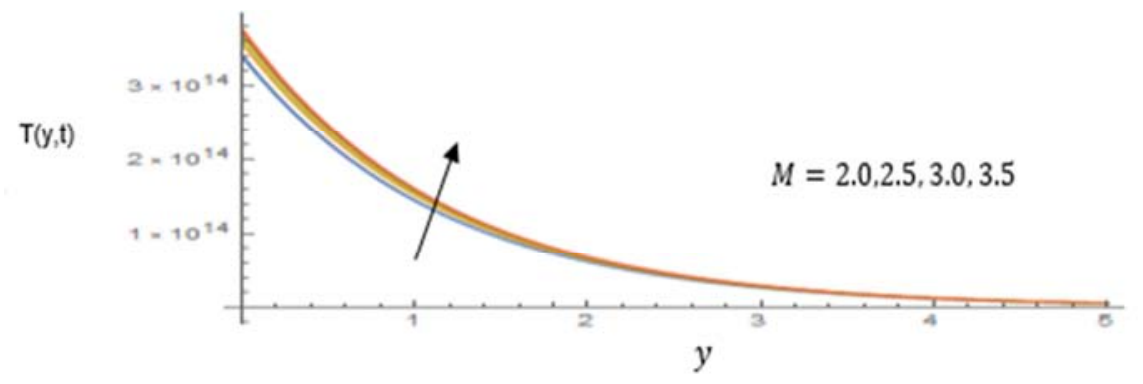

Figure 11. Temperature Profile with Variation of Magnetic Field Parameter $M$ for $H=0.5, P_{r}=0.71, G_{r}=15, G_{c}=10, S_{c}=0.22, K r=2, E c=0.01, R=$ $0.1, N=0.1, \alpha=10, \omega=1, t=1, \varepsilon=1$.

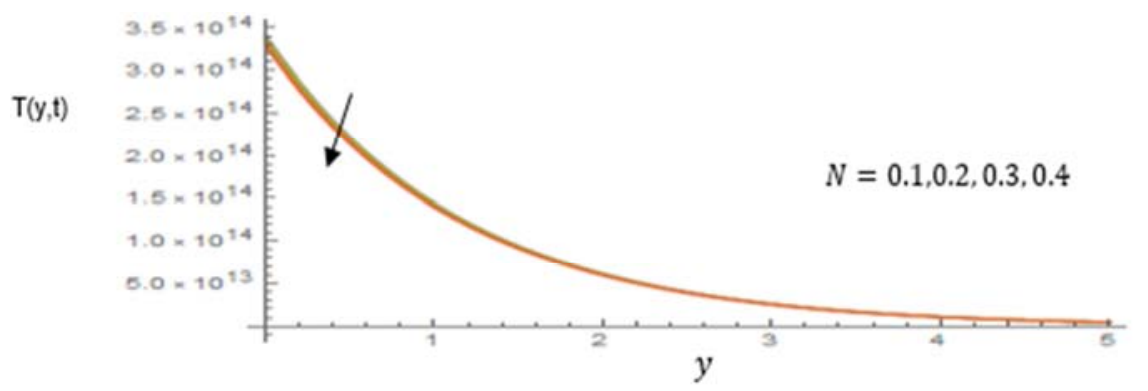

Figure 12. Temperature Profile with Variation of Porosity Parameter $N$ for $M=2, H=0.5, P_{r}=0.71, G_{r}=15, G_{c}=10, S_{c}=0.22, K r=2, E c=0.01, R=$ $0.1, \alpha=10, \omega=1, t=1, \varepsilon=1$.

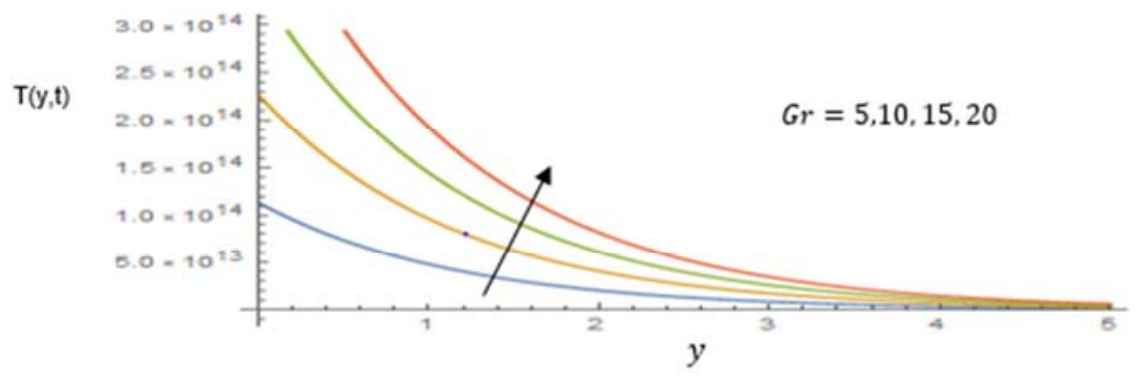

Figure 13. Temperature Profile with Variation of Grashof Temperature Parameter Gr for $M=2, H=0.5, P_{r}=0.71, G_{c}=10, S_{c}=0.22, K r=2, E c=$ $0.01, R=0.1, N=0.1, \alpha=10, \omega=1, t=1, \varepsilon=1$.

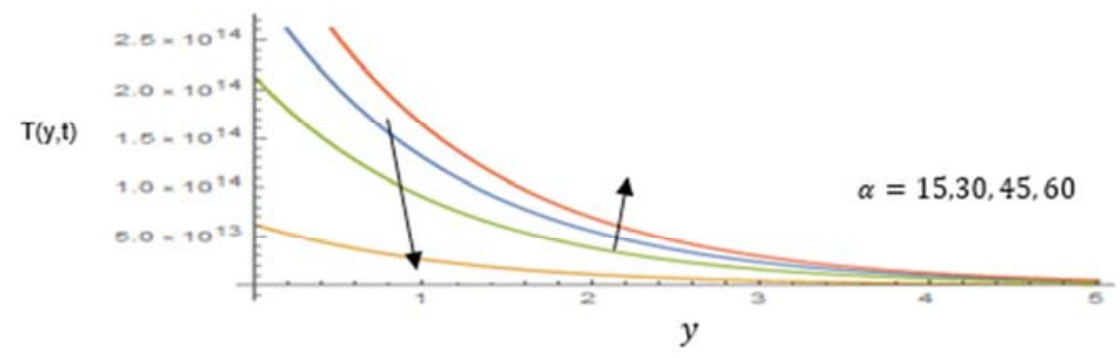

Figure 14. Temperature Profile with Variation of angle of inclination Parameter $\alpha$ for $M=2, H=0.5, P_{r}=0.71, G_{r}=15, G_{c}=10, S_{c}=0.22, K r=2, E c=$ $0.01, R=0.1, N=0.1, \omega=1, t=1, \varepsilon=1$. 


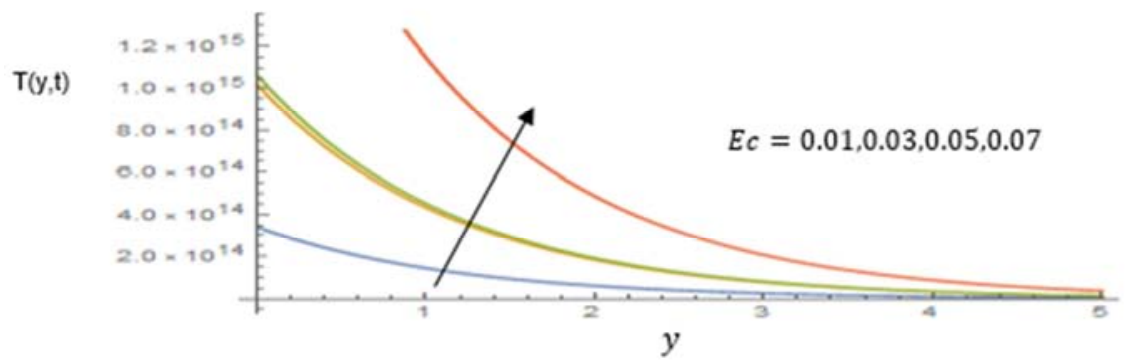

Figure 15. Temperature Profile with Variation of Viscous Dissipation Parameter Ec for $M=2, S=0.5, P_{r}=0.71, G_{r}=15, G_{c}=10, S_{c}=0.22, K r=2, R=$ $0.1, N=0.1, \alpha=10, \omega=1, t=1, \varepsilon=1$

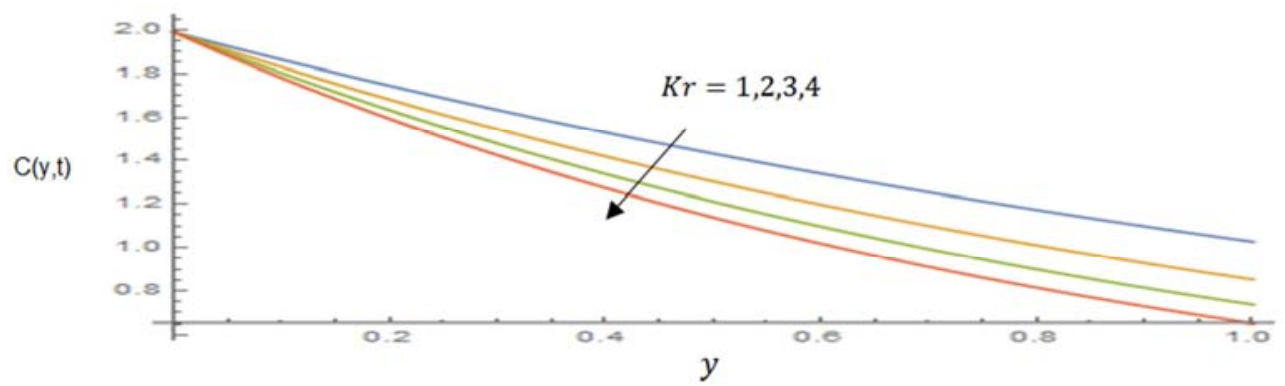

Figure 16. Concentration Profile with Variation of Chemical Reaction Parameter Kr for $S c=0.22, \omega=1, t=1, \varepsilon=1$.

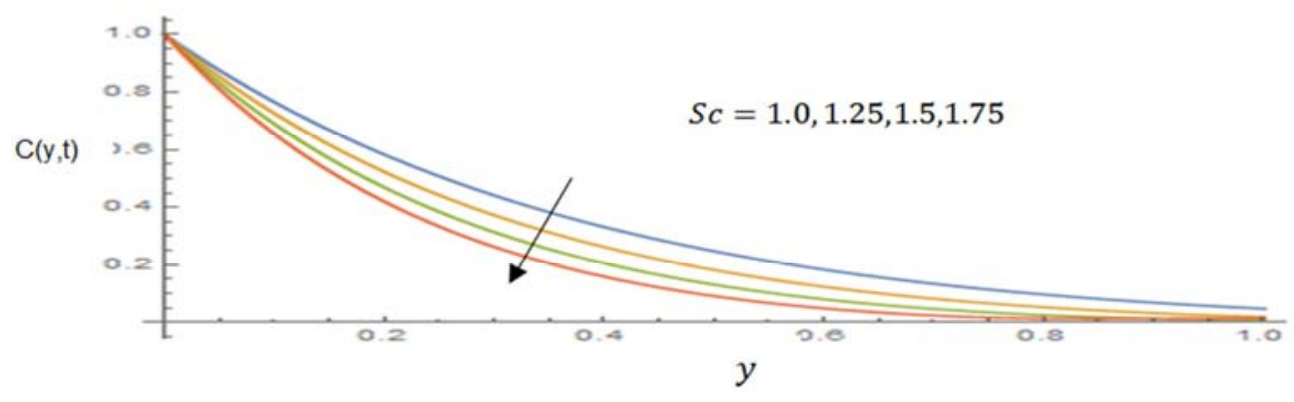

Figure 17. Concentration Profile with Variation of Schmidt number Parameter $S c$ for $K r=2, \omega=1, t=1, \varepsilon=1$.

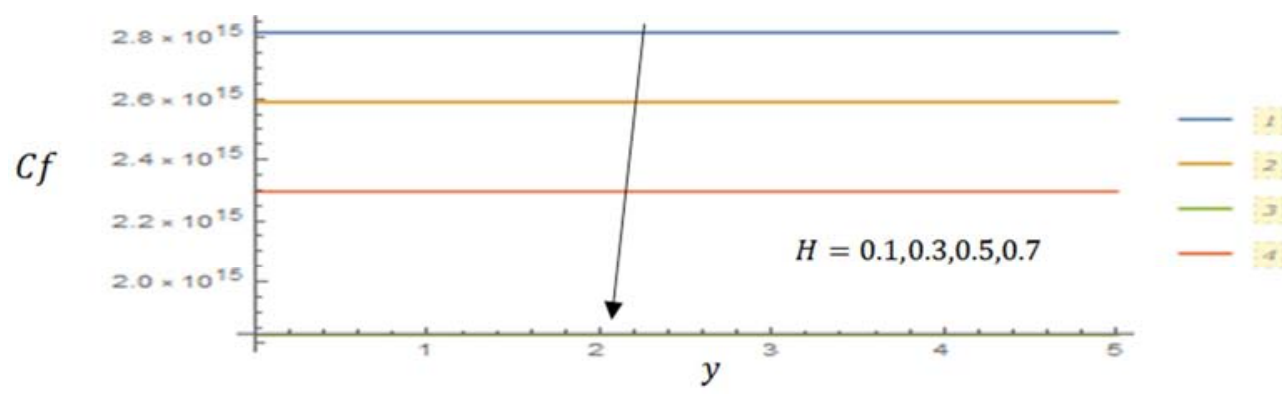

Figure 18. Skin Friction Profile with Variation of Heat Source Parameter $H$ for $M=2, P_{r}=0.71, G_{r}=15, G_{c}=10, S_{c}=0.22, K r=2, E c=0.01, R=$ $0.1, N=0.1, \alpha=10, \omega=1, t=1, \varepsilon=1$.

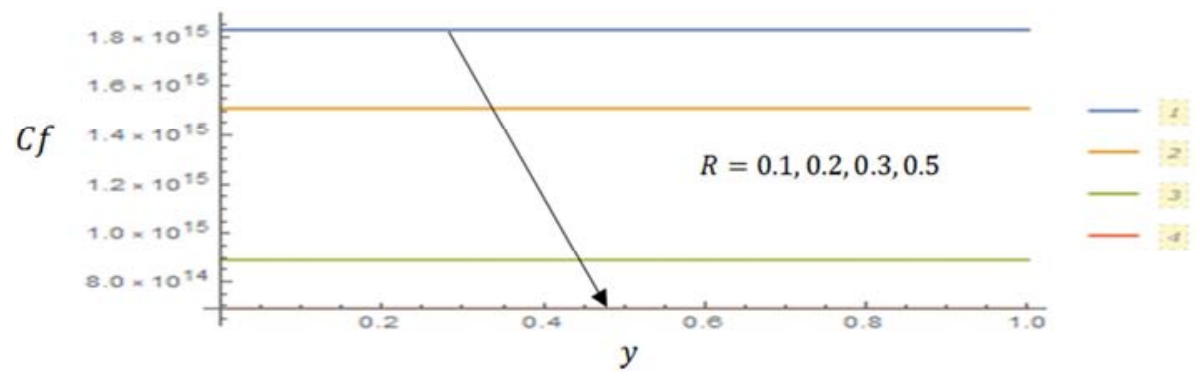

Figure 19. Skin Friction Profile with Variation of Radiation Parameter $R$ for $M=2, H=0.5, P_{r}=0.71, G_{r}=15, G_{c}=10, S_{c}=0.22, K r=2, E c=$ $0.01, N=0.1, \alpha=10, \omega=1, t=1, \varepsilon=1$. 


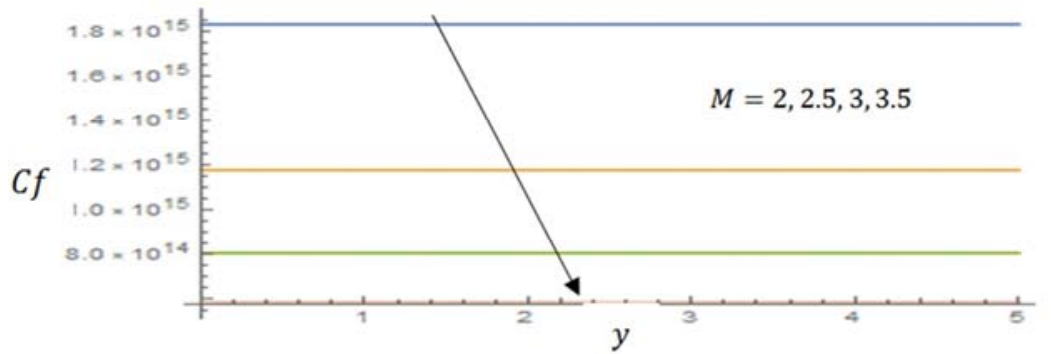

Figure 20. Skin Friction Profile with Variation of Magnetic Field Parameter $M$ for $H=0.5, P_{r}=0.71, G_{r}=15, G_{c}=10, S_{c}=0.22, K r=2, E c=0.01, R=$ $0.1, N=0.1, \alpha=10, \omega=1, t=1, \varepsilon=1$.

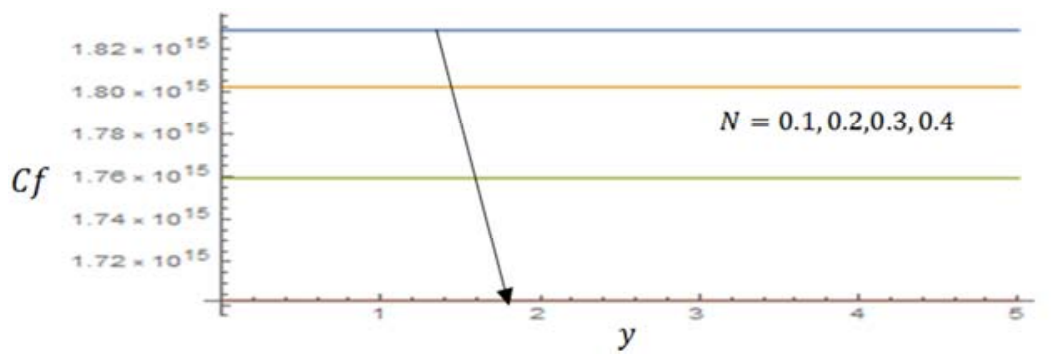

Figure 21. Skin Friction Profile with Variation of Porosity Parameter $N$ for $M=2, H=0.5, P_{r}=0.71, G_{r}=15, G_{c}=10, S_{c}=0.22, K r=2, E c=0.01, R=$ $0.1, \alpha=10, \omega=1, t=1, \varepsilon=1$.

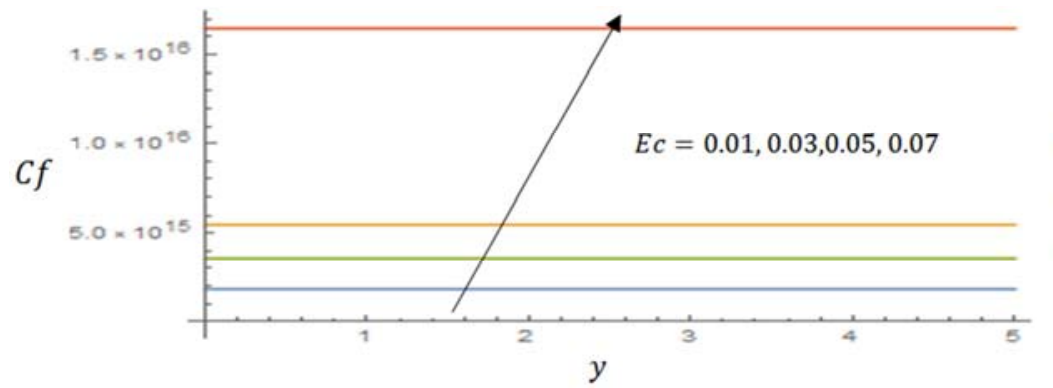

Figure 22. Skin Friction Profile with Variation of Viscous Dissipation Parameter Ec for $M=2, H=0.5, P_{r}=0.71, G_{r}=15, G_{c}=10, S_{c}=0.22, K r=$ $2, R=0.1, N=0.1, \alpha=10, \omega=1, t=1, \varepsilon=1$.

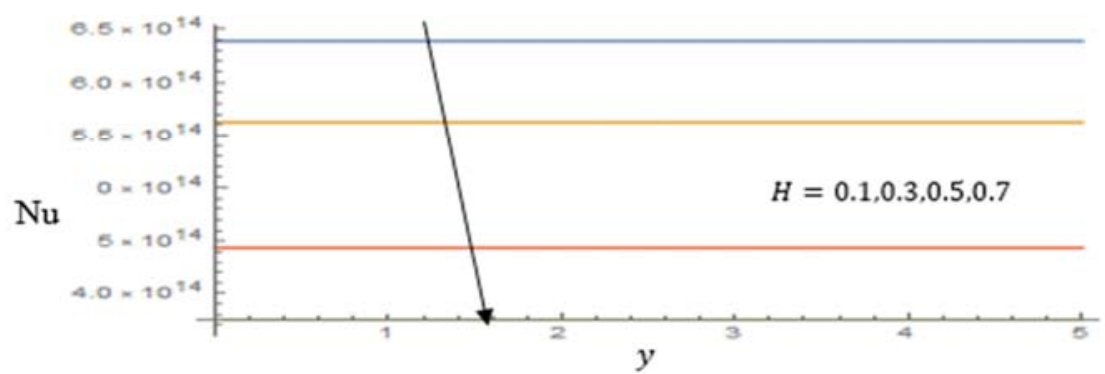

Figure 23. Heat Transfer Profile with Variation of Heat Source Parameter for $M=2, P_{r}=0.71, G_{r}=15, G_{c}=10, S_{c}=0.22, K r=2, E c=0.01, R=$ $0.1, N=0.1, \alpha=10, \omega=1, t=1, \varepsilon=1$.

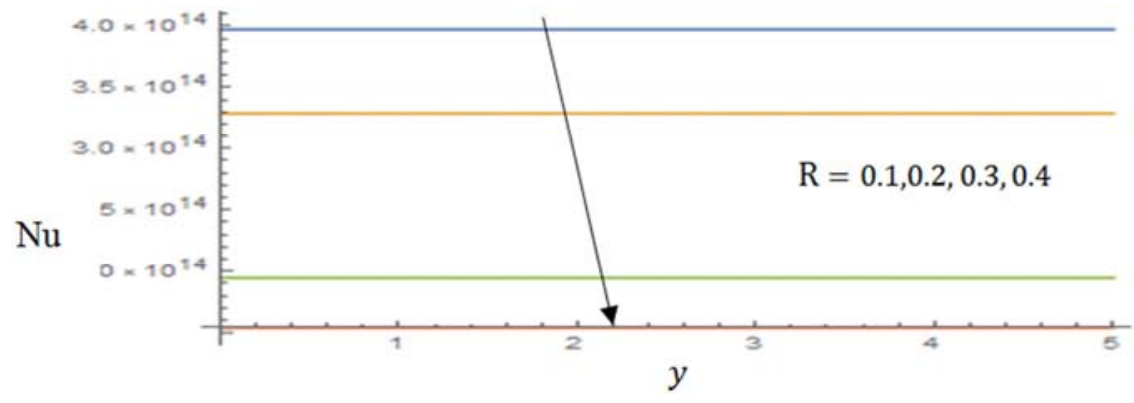

Figure 24. Heat Transfer Profile with Variation of Radiation Parameter for $M=2, H=0.5, P_{r}=0.71, G_{r}=15, G_{c}=10, S_{c}=0.22, K r=2, E c=0.01, N=$ $0.1, \alpha=10, \omega=1, t=1, \varepsilon=1$. 


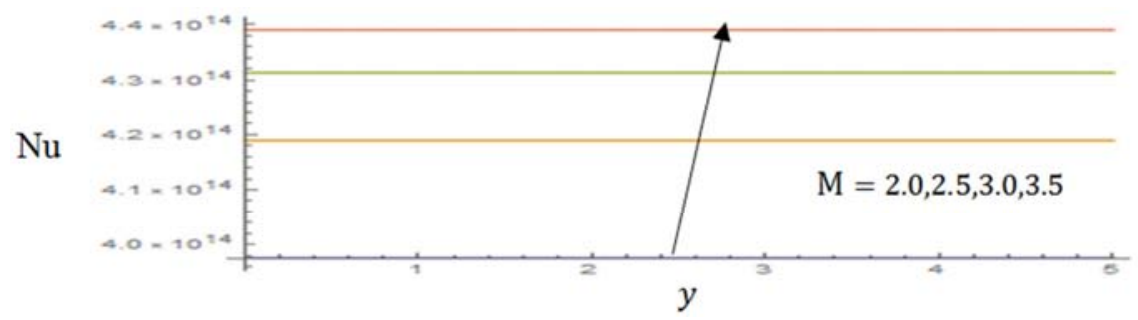

Figure 25. Heat Transfer Profile with Variation of Magnetic Field Parameter for $H=0.5, P_{r}=0.71, G_{r}=15, G_{c}=10, S_{c}=0.22, K r=2, E c=0.01, R=$ $0.1, N=0.1, \alpha=10, \omega=1, t=1, \varepsilon=1$.

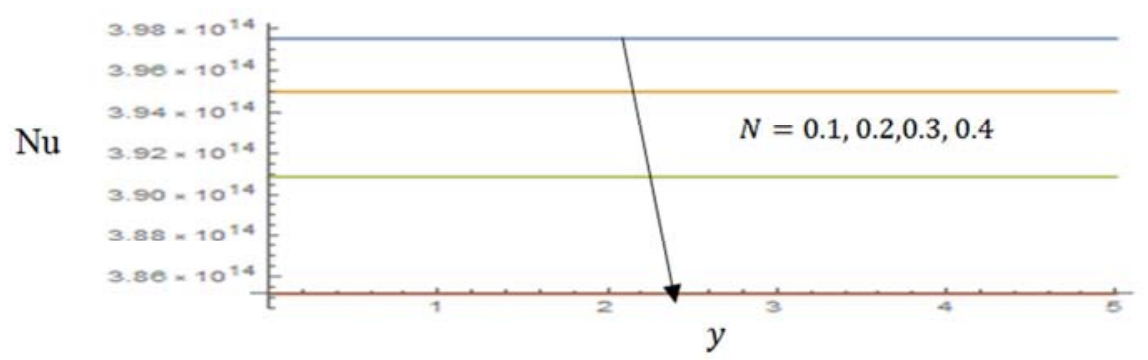

Figure 26. Heat Transfer Profile with Variation of Porosity Parameter for $M=2, H=0.5, P_{r}=0.71, G_{r}=15, G_{c}=10, S_{c}=0.22, K r=2, E c=0.01, R=$ $0.1, \alpha=10, \omega=1, t=1, \varepsilon=1$.

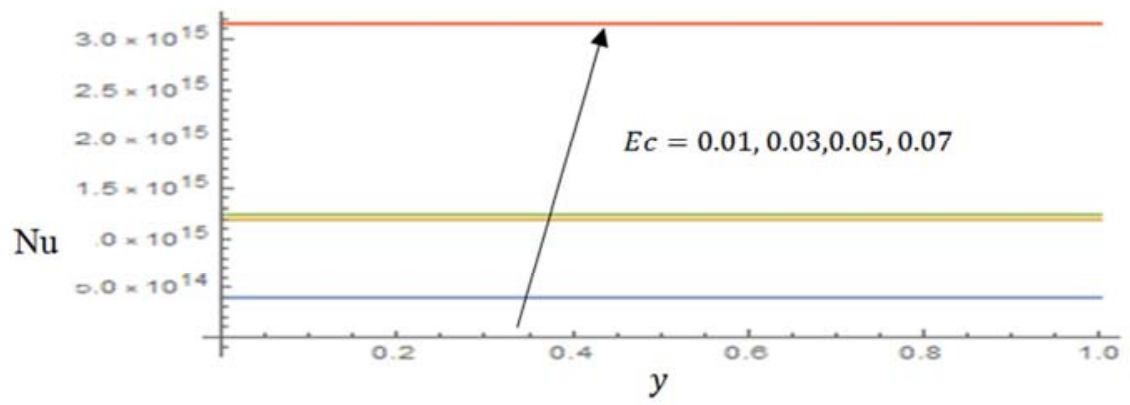

Figure 27. Heat Transfer Profile with Variation of Viscous Dissipation Parameter Ec for $M=2, H=0.5, P_{r}=0.71, G_{r}=15, G_{c}=10, S_{c}=0.22, K r=$ $2, R=0.1, N=0.1, \alpha=10, \omega=1, t=1, \varepsilon=1$.

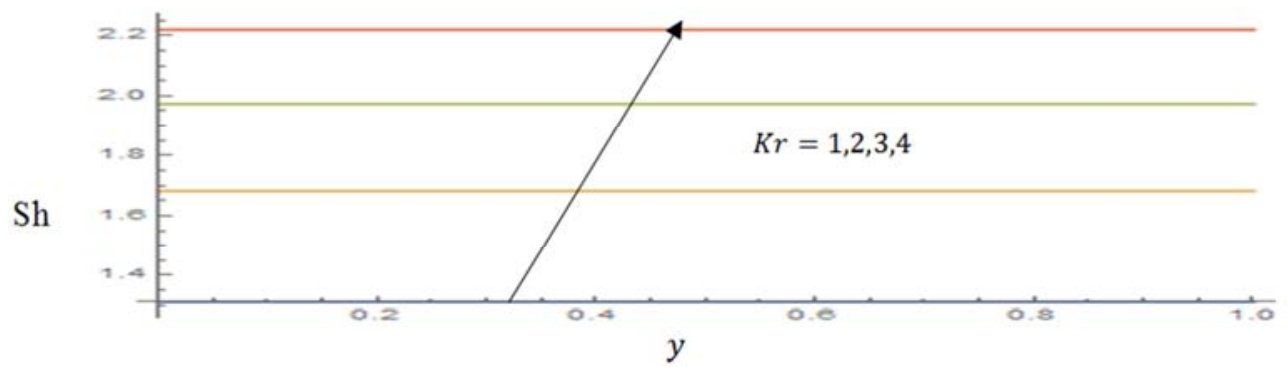

Figure 28. Mass Transfer Profile with Variation of Chemical Reaction Parameter Kr for $S c=0.22, \omega=1, t=1, \varepsilon=1$.

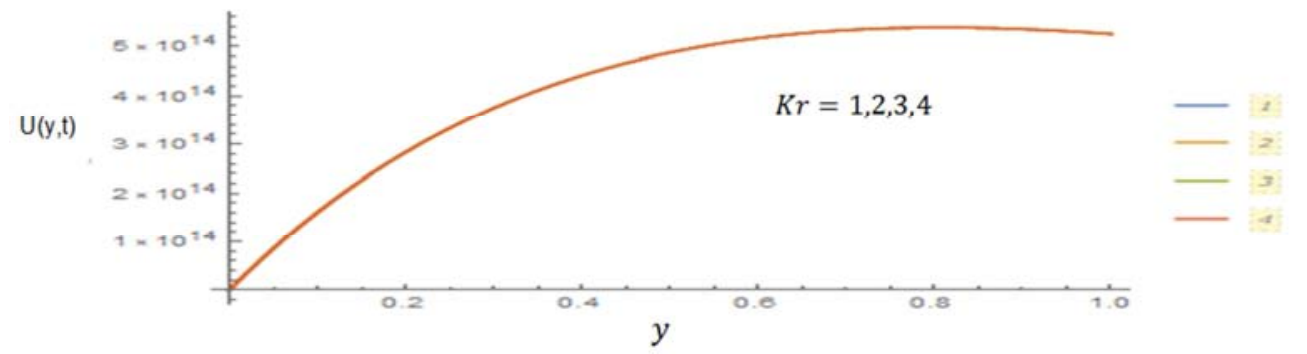

Figure 29. Velocity Profile with Variation of Chemical Reaction Parameter Kr for $M=2, H=0.5, P_{r}=0.71, G_{r}=15, G_{c}=10, S_{c}=0.22, E c=0.01, R=$ $0.1, N=0.1, \alpha=10, \omega=1, t=1, \varepsilon=1$. 


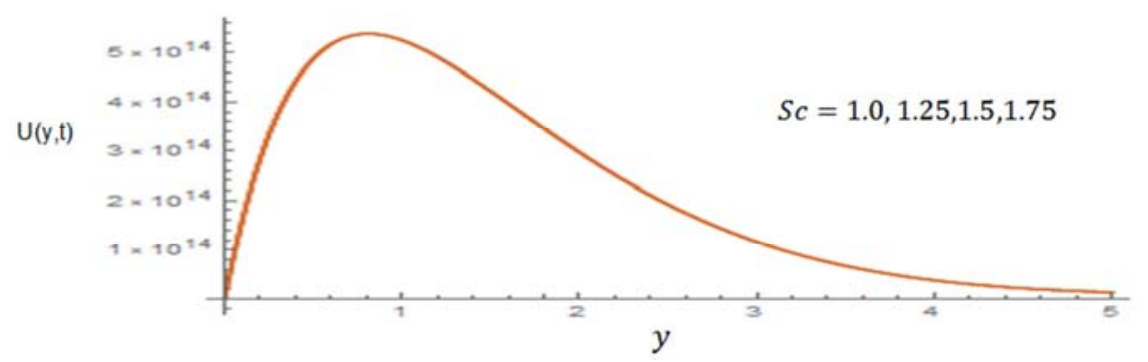

Figure 30. Velocity Profile with Variation of Schmidt Number Parameter $S c$ for $M=2, H=0.5, P_{r}=0.71, G_{r}=15, G_{c}=10, E c=0.01, R=0.1, N=$ $0.1, \alpha=10, \omega=1, t=1, \varepsilon=1$.

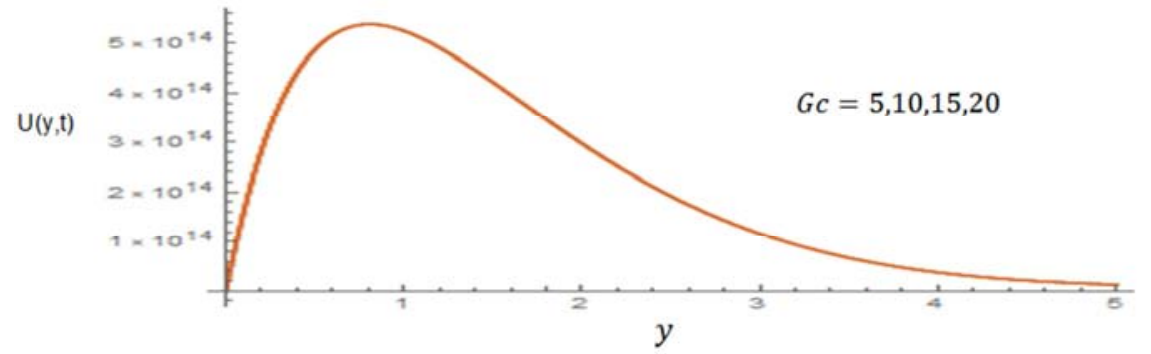

Figure 31. Velocity Profile with Variation of Grashof diffusion Parameter Gc for $M=2, H=0.5, P_{r}=0.71, G_{r}=15, G_{c}=10, E c=0.01, R=0.1, N=$ $0.1, \alpha=10, \omega=1, t=1, \varepsilon=1$.

\section{Conclusion}

Radiation, Viscous dissipation and heat source effects on MHD free convection flow over an inclined porous surface are summarized as follows,

a) Increased Magnetic field reduces the velocity profile, increases the temperature, decreases skin friction and increases heat transfer profile.

b) Increase in radiation reduces the velocity, temperature, Skin friction and heat transfer profile.

c) An increase in the heat source caused a mixed effect on the velocity, decreases the temperature near the plate, decreases skin friction profile and heat transfer profile. d) Increase in porosity reduces the velocity, temperature, Skin friction and heat transfer profile.

e) Increase in the angle of inclination of the porous plate initially reduces the velocity and temperature profile but later increases the velocity and temperature profile, hence having a mixed effect on both velocity and temperature.

f) Increase in viscous dissipation increases velocity, temperature, Skin friction and heat transfer profile.

g) From the research so far parameters such Grashof concentration number (Gc), Schmidt number (Sc) and Chemical reaction $(\mathrm{Kr})$ had no effect on the velocity profile and temperature profile.

\section{Appendix}

$$
\begin{gathered}
M^{2}=\frac{v \mu^{2} \delta_{c} H_{O}^{\prime 2}}{\rho v_{o}^{\prime 2}} ; \mathrm{N}^{2}=\frac{v^{2}}{K v_{o}^{\prime \prime}} ; G_{r}=\frac{g v B_{T} \theta\left(T_{w}{ }^{\prime}-T_{\infty}\right)}{V_{O} v_{o}^{\prime 2}} ; G_{c}=\frac{g v B_{C} C\left(C_{w}{ }^{\prime}-C_{\infty}\right)}{V_{O} v_{o}^{\prime 2}} ; P_{r}=\frac{\mu C_{p}}{k} ; R^{2}=\frac{16 v \delta^{\prime} T_{O}^{3}}{3 k^{\prime}} ; \\
E_{C}=\frac{V_{O}^{2}}{C_{p}\left(T_{w}^{\prime}-T_{\infty}\right)} ; S_{c}=\frac{v}{D} ; m_{1}=\frac{S_{c}+\sqrt{ }\left(S_{c}{ }^{2}+4 S_{c} K_{r}\right)}{2} ; m_{2}=\frac{-P_{r}-\sqrt{ }\left(P_{r}{ }^{2}+4 H P r\left(1+R^{2}\right)\right)}{2\left(1+R^{2}\right)}=m_{4} ; \\
m_{3}=\frac{1+\sqrt{ }\left(1^{2}+4\left(\mathrm{~N}^{2}+M^{2}\right)\right)}{2}=m_{5} ; m_{6}=\frac{S_{c}+\sqrt{ }\left(S_{c}{ }^{2}+4\left(S_{c} K_{r}+S_{c} i \omega\right)\right)}{2} \\
m_{7}=\frac{P_{r}+\sqrt{ }\left(P_{r}{ }^{2}-4\left(H-P_{r} i \omega\right)\left(1+R^{2}\right)\right)}{2\left(1+R^{2}\right)}=m_{9} ; m_{8}=\frac{1+\sqrt{\left(1^{2}+4\left(\mathrm{~N}^{2}+M^{2}+i \omega\right)\right)}}{2}=m_{10} \\
\mathrm{~A} 2=\frac{\mathrm{A} 1=\mathrm{A} 2+\mathrm{A} 3}{\mathrm{~m} 2^{2}+\mathrm{m} 2-\left(M^{2}+N^{2}\right)} ; \\
\mathrm{A} 3=\frac{\mathrm{GcCos}[\alpha]}{\mathrm{m} 2^{2}+\mathrm{m} 2-\left(M^{2}+N^{2}\right)} ;
\end{gathered}
$$




$$
\begin{aligned}
& \mathrm{A} 4=\mathrm{A} 5+\mathrm{A} 6+\mathrm{A} 7-\mathrm{A} 8-\mathrm{A} 9+\mathrm{A} 10+\mathrm{A} 11-\mathrm{A} 12-\mathrm{A} 13 ; \\
& \mathrm{A} 5=\frac{\operatorname{Prm}^{2} \mathrm{~A} 1^{2}}{4\left(1+R^{2}\right) \mathrm{m} 3^{2}-2 \operatorname{Prm} 3+H \operatorname{Pr}} ; \\
& \mathrm{A} 6=\frac{\operatorname{Prm}^{2} \mathrm{~A}^{2}}{4\left(1+R^{2}\right) \mathrm{m} 2^{2}-2 \operatorname{Prm} 2+H \operatorname{Pr}} ; \\
& \mathrm{A} 7=\frac{\operatorname{Prm}^{2} \mathrm{~A}^{2}}{4\left(1+R^{2}\right) \mathrm{m} 1^{2}-2 \operatorname{Prm} 1+H \operatorname{Pr}} ; \\
& \mathrm{A} 8=\frac{2 \operatorname{Prm} 2 \mathrm{~m} 3 \mathrm{~A} 1 \mathrm{~A} 2}{\left(1+R^{2}\right)(\mathrm{m} 2+\mathrm{m} 3)^{2}-\operatorname{Pr}(\mathrm{m} 2+\mathrm{m} 3)+H \operatorname{Pr}} \text {; } \\
& \mathrm{A} 9=\frac{2 \operatorname{Prm} 1 \mathrm{~m} 2 \mathrm{~A} 1 \mathrm{~A} 3}{\left(1+R^{2}\right)(\mathrm{m} 1+\mathrm{m} 3)^{2}-\operatorname{Pr}(\mathrm{m} 1+\mathrm{m} 3)+H \operatorname{Pr}} \text {; } \\
& \mathrm{A} 10=\frac{2 \operatorname{Prm} 1 \mathrm{~m} 2 \mathrm{~A} 2 \mathrm{~A} 3}{\left(1+R^{2}\right)(\mathrm{m} 1+\mathrm{m} 2)^{2}-\operatorname{Pr}(\mathrm{m} 1+\mathrm{m} 2)+H \operatorname{Pr}} ; \\
& \mathrm{A} 11=\frac{\operatorname{Prm}^{2} \mathrm{~A}^{2}}{\left(1+R^{2}\right) \mathrm{m}^{2}-\operatorname{Prm} 3+H \operatorname{Pr}} ; \\
& \mathrm{A} 12=\frac{\operatorname{Prm}^{2} \mathrm{~A} 2^{2}}{\left(1+R^{2}\right) \mathrm{m} 2^{2}-\operatorname{Prm} 2+H \operatorname{Pr}} ; \\
& \mathrm{A} 13=\frac{\operatorname{Prm}^{2} \mathrm{~A} 3^{2}}{\left(1+R^{2}\right) \mathrm{m} 1^{2}-\operatorname{Prm} 1+H \operatorname{Pr}} ; \\
& \mathrm{A} 15=\frac{\operatorname{GrA} 4 \operatorname{Cos}[\alpha]}{\mathrm{m} 4^{2}-\mathrm{m} 4+\left(M^{2}+N^{2}\right)} ; \\
& \mathrm{A} 16=\frac{\operatorname{GrA} 5 \operatorname{Cos}[\alpha]}{4 \mathrm{~m} 3^{2}+2 \mathrm{~m} 3-\left(M^{2}+N^{2}\right)} ; \\
& \mathrm{A} 17=\frac{\operatorname{GrA6} \operatorname{Cos}[\alpha]}{4 \mathrm{~m} 2^{2}+2 \mathrm{~m} 2-\left(M^{2}+N^{2}\right)} ; \\
& \mathrm{A} 18=\frac{\operatorname{GrA7} \operatorname{Cos}[\alpha]}{4 \mathrm{~m} 1^{2}+2 \mathrm{~m} 1-\left(M^{2}+N^{2}\right)} ; \\
& \mathrm{A} 19=\frac{\operatorname{GrA8Cos}[\alpha]}{(\mathrm{m} 2+\mathrm{m} 3)^{2}+(\mathrm{m} 2+\mathrm{m} 3)-\left(M^{2}+N^{2}\right)} ; \\
& \mathrm{A} 20=\frac{\mathrm{GrA9 \operatorname {Cos } [ \alpha ]}}{(\mathrm{m} 1+\mathrm{m} 3)^{2}+(\mathrm{m} 1+\mathrm{m} 3)-\left(M^{2}+N^{2}\right)} ; \\
& \mathrm{A} 21=\frac{\mathrm{GrA10} \operatorname{Cos}[\alpha]}{(\mathrm{m} 1+\mathrm{m} 2)^{2}+(\mathrm{m} 1+\mathrm{m} 2)-\left(M^{2}+N^{2}\right)} \\
& \mathrm{A} 22=\frac{\operatorname{GrA} 11 \operatorname{Cos}[\alpha]}{\mathrm{m} 3^{2}+\mathrm{m} 3-\left(M^{2}+N^{2}\right)} \\
& \mathrm{A} 23=\frac{\operatorname{GrA} 12 \operatorname{Cos}[\alpha]}{\mathrm{m} 2^{2}+2 \mathrm{~m} 2-\left(M^{2}+N^{2}\right)} \\
& \mathrm{A} 24=\frac{\operatorname{GrA} 13 \operatorname{Cos}[\alpha]}{4 \mathrm{~m} 1^{2}+2 \mathrm{~m} 1-\left(M^{2}+N^{2}\right)} \\
& \mathrm{A} 25=-\mathrm{A} 26
\end{aligned}
$$




$$
\begin{aligned}
& \mathrm{A} 26=\frac{\mathrm{m} 1 \mathrm{Sc}}{\mathrm{m} 1^{2}-\mathrm{Scm} 1-(\mathrm{ScKr}+i \omega \mathrm{Sc})} \\
& \mathrm{A} 27=-\mathrm{A} 28 \\
& \mathrm{~A} 28=\frac{\operatorname{Prm} 2}{\left(1+R^{2}\right) \mathrm{m}^{2}-\operatorname{Prm} 2+(H \operatorname{Pr}-\operatorname{Pr} i \omega)} \\
& \mathrm{A} 29=\mathrm{A} 30+\mathrm{A} 31+\mathrm{A} 32+\mathrm{A} 33-\mathrm{A} 34+\mathrm{A} 35+\mathrm{A} 36 \\
& \mathrm{~A} 30=\frac{\mathrm{GrA27 \operatorname {Cos }}[\alpha]}{\mathrm{m} 7^{2}-\mathrm{m} 7-\left(M^{2}+N^{2}+i \omega\right)} \\
& \mathrm{A} 31=\frac{\mathrm{GrA} 28 \operatorname{Cos}[\alpha]}{\mathrm{m} 2^{2}-\mathrm{m} 2-\left(M^{2}+N^{2}+i \omega\right)} \\
& \mathrm{A} 32=\frac{\mathrm{GcA} 25 \operatorname{Cos}[\alpha]}{\mathrm{m} 6^{2}-\mathrm{m} 6-\left(M^{2}+N^{2}+i \omega\right)} \\
& \mathrm{A} 33=\frac{\mathrm{GcA} 26 \operatorname{Cos}[\alpha]}{\mathrm{m} 1^{2}-\mathrm{m} 1-\left(M^{2}+N^{2}+i \omega\right)} \\
& \mathrm{A} 34=\frac{\mathrm{m} 3 \mathrm{~A} 1}{\mathrm{~m} 3^{2}-\mathrm{m} 3-\left(M^{2}+N^{2}+i \omega\right)} \\
& \mathrm{A} 35=\frac{\mathrm{m} 2 \mathrm{~A} 2}{\mathrm{~m} 2^{2}-\mathrm{m} 2-\left(M^{2}+N^{2}+i \omega\right)} \\
& \mathrm{A} 36=\frac{\mathrm{m} 1 \mathrm{~A} 3}{\mathrm{~m} 1^{2}-\mathrm{m} 1-\left(M^{2}+N^{2}+i \omega\right)} \\
& \mathrm{A} 37=1 \\
& \mathrm{~A} 38=\frac{\operatorname{Prm} 4 \mathrm{~A} 4}{\left(1+R^{2}\right) \mathrm{m} 4^{2}-\operatorname{Prm} 4+(H \operatorname{Pr}-\operatorname{Pr} i \omega)} \\
& \mathrm{A} 39=\frac{(2 \mathrm{Prm} 3)(\mathrm{m} 3 \mathrm{~A} 1 \mathrm{~A} 34+\mathrm{A} 5)}{4\left(1+R^{2}\right) \mathrm{m}^{2}-\operatorname{Prm} 3+(H \operatorname{Pr}-\operatorname{Pr} i \omega)} \\
& \mathrm{A} 40=\frac{(2 \operatorname{Prm} 2)(\mathrm{m} 2 \mathrm{~A} 31 \mathrm{~A} 2+\mathrm{m} 2 \mathrm{~A} 2 \mathrm{~A} 35+\mathrm{A} 6)}{4\left(1+R^{2}\right) \mathrm{m}^{2}-\operatorname{Prm} 2+(H \operatorname{Pr}-\operatorname{Pri} \omega)} \\
& \mathrm{A} 41=\frac{(2 \operatorname{Prm} 1)(\mathrm{m} 1 \mathrm{~A} 3 \mathrm{~A} 33+\mathrm{m} 1 \mathrm{~A} 3 \mathrm{~A} 36+\mathrm{A} 7)}{4\left(1+R^{2}\right) \mathrm{m} 1^{2}-\operatorname{Prm} 1+(H \operatorname{Pr}-\operatorname{Pr} i \omega)} \\
& \mathrm{A} 42=\frac{\operatorname{Pr}(\mathrm{m} 2+\mathrm{m} 3) \mathrm{A} 8+(2 \mathrm{~m} 2 \mathrm{~m} 3)(\mathrm{A} 1 \mathrm{~A} 31+\mathrm{A} 1 \mathrm{~A} 35+\mathrm{A} 2 \mathrm{~A} 34)}{4\left(1+R^{2}\right)(\mathrm{m} 2+\mathrm{m} 3)^{2}-\operatorname{Pr}(\mathrm{m} 2+\mathrm{m} 3)+(H \operatorname{Pr}-\operatorname{Pr} i \omega)} \\
& \mathrm{A} 43=\frac{\operatorname{Pr}(\mathrm{m} 1+\mathrm{m} 3) \mathrm{A} 9+(2 \mathrm{~m} 1 \mathrm{~m} 3)(\mathrm{A} 1 \mathrm{~A} 33+\mathrm{A} 1 \mathrm{~A} 36+\mathrm{A} 3 \mathrm{~A} 34)}{4\left(1+R^{2}\right)(\mathrm{m} 1+\mathrm{m} 3)^{2}-\operatorname{Pr}(\mathrm{m} 1+\mathrm{m} 3)+(\text { Hr }-\operatorname{Pri} \omega)} \\
& \mathrm{A} 44=\frac{\operatorname{Pr}(\mathrm{m} 1+\mathrm{m} 2) \mathrm{A} 10+(2 \mathrm{~m} 1 \mathrm{~m} 2)(\mathrm{A} 2 \mathrm{~A} 33+\mathrm{A} 2 \mathrm{~A} 36+\mathrm{A} 3 \mathrm{~A} 31+\mathrm{A} 3 \mathrm{~A} 35)}{4\left(1+R^{2}\right)(\mathrm{m} 1+\mathrm{m} 2)^{2}-\operatorname{Pr}(\mathrm{m} 1+\mathrm{m} 2)+(H \operatorname{Pr}-\operatorname{Pr} i \omega)} \\
& \mathrm{A} 45=\frac{\operatorname{Pr}\left(\mathrm{m} 3 \mathrm{~A} 11+M^{2} \mathrm{~A} 34\right)}{\left(1+R^{2}\right) \mathrm{m} 3^{2}-\operatorname{Prm} 3+(H \operatorname{Pr}-\operatorname{Pr} i \omega)} \\
& \mathrm{A} 46=\frac{\operatorname{Pr}\left(\mathrm{m} 2 \mathrm{~A} 12+M^{2}(\mathrm{~A} 31+\mathrm{A} 35)\right)}{\left(1+R^{2}\right) \mathrm{m}^{2}-\operatorname{Prm} 3+(H \operatorname{Pr}-\operatorname{Pr} i \omega)} \\
& \mathrm{A} 47=\frac{\operatorname{Pr}\left(\mathrm{m} 1 \mathrm{~A} 13+M^{2}(\mathrm{~A} 33+\mathrm{A} 36)\right)}{\left(1+R^{2}\right) \mathrm{m}^{2}-\operatorname{Prm} 1+(H \operatorname{Pr}-\operatorname{Pr} i \omega)}
\end{aligned}
$$




$$
\begin{aligned}
& \mathrm{A} 48=\frac{2 \operatorname{Prm} 3 \mathrm{~m} 8 \mathrm{~A} 1 \mathrm{~A} 29}{\left(1+R^{2}\right)(\mathrm{m} 3+\mathrm{m} 8)^{2}-\operatorname{Pr}(\mathrm{m} 3+\mathrm{m} 8)+(H \operatorname{Pr}-\operatorname{Pr} \omega)} \\
& \mathrm{A} 49=\frac{2 \operatorname{Prm} 3 \mathrm{~m} 7 \mathrm{~A} 1 \mathrm{~A} 30}{\left(1+R^{2}\right)(\mathrm{m} 3+\mathrm{m} 7)^{2}-\operatorname{Pr}(\mathrm{m} 3+\mathrm{m} 7)+(H \operatorname{Pr}-\operatorname{Pr} i \omega)} \\
& \mathrm{A} 50=\frac{2 \operatorname{Prm} 3 \mathrm{~m} 6 \mathrm{~A} 1 \mathrm{~A} 32}{\left(1+R^{2}\right)(\mathrm{m} 3+\mathrm{m} 6)^{2}-\operatorname{Pr}(\mathrm{m} 3+\mathrm{m} 6)+(H \operatorname{Pr}-\operatorname{Pr} i \omega)} \\
& \mathrm{A} 51=\frac{2 \operatorname{Prm} 2 \mathrm{~m} 8 \mathrm{~A} 2 \mathrm{~A} 29}{\left(1+R^{2}\right)(\mathrm{m} 2+\mathrm{m} 8)^{2}-\operatorname{Pr}(\mathrm{m} 2+\mathrm{m} 8)+(H \operatorname{Pr}-\operatorname{Pr} i \omega)} \\
& \mathrm{A} 52=\frac{2 \operatorname{Prm} 2 \mathrm{~m} 7 \mathrm{~A} 2 \mathrm{~A} 30}{\left(1+R^{2}\right)(\mathrm{m} 2+\mathrm{m} 7)^{2}-\operatorname{Pr}(\mathrm{m} 2+\mathrm{m} 7)+(H \operatorname{Pr}-\operatorname{Pr} i \omega)} \\
& \mathrm{A} 53=\frac{2 \operatorname{Prm} 2 \mathrm{~m} 6 \mathrm{~A} 2 \mathrm{~A} 32}{\left(1+R^{2}\right)(\mathrm{m} 2+\mathrm{m} 6)^{2}-\operatorname{Pr}(\mathrm{m} 2+\mathrm{m} 6)+(H \operatorname{Pr}-\operatorname{Pr} i \omega)} \\
& \mathrm{A} 54=\frac{2 \operatorname{Prm} 1 \mathrm{~m} 8 \mathrm{~A} 3 \mathrm{~A} 29}{\left(1+R^{2}\right)(\mathrm{m} 1+\mathrm{m} 8)^{2}-\operatorname{Pr}(\mathrm{m} 1+\mathrm{m} 8)+(H \operatorname{Pr}-\operatorname{Pr} \omega)} \\
& \mathrm{A} 55=\frac{2 \operatorname{Prm} 1 \mathrm{~m} 7 \mathrm{~A} 3 \mathrm{~A} 30}{\left(1+R^{2}\right)(\mathrm{m} 1+\mathrm{m} 7)^{2}-\operatorname{Pr}(\mathrm{m} 1+\mathrm{m} 7)+(H \operatorname{Pr}-\operatorname{Pr} i \omega)} \\
& \mathrm{A} 56=\frac{2 \operatorname{Prm} 1 \mathrm{~m} 6 \mathrm{~A} 3 \mathrm{~A} 32}{\left(1+R^{2}\right)(\mathrm{m} 1+\mathrm{m} 6)^{2}-\operatorname{Pr}(\mathrm{m} 1+\mathrm{m} 6)+(H \operatorname{Pr}-\operatorname{Pr} i \omega)} \\
& \mathrm{A} 57=\frac{M^{2} \operatorname{PrA} 29}{\left(1+R^{2}\right) \mathrm{m} 1^{2}-\operatorname{Prm} 1+(H \operatorname{Pr}-\operatorname{Pr} i \omega)} \\
& \mathrm{A} 58=\frac{M^{2} \operatorname{PrA} 30}{\left(1+R^{2}\right) \mathrm{m}^{2}-\operatorname{Prm} 7+(H \operatorname{Pr}-\operatorname{Pr} i \omega)} \\
& \mathrm{A} 59=\frac{M^{2} \operatorname{PrA} 32}{\left(1+R^{2}\right) \mathrm{m}^{2}-\operatorname{Prm} 6+(H \operatorname{Pr}-\operatorname{Pr} i \omega)} \\
& \mathrm{A} 60=\mathrm{A} 61+\mathrm{A} 62-\mathrm{A} 63+\mathrm{A} 64+\mathrm{A} 65-\mathrm{A} 66+\mathrm{A} 67+\mathrm{A} 68+\mathrm{A} 69-\mathrm{A} 70-\mathrm{A} 71-\mathrm{A} 72+\mathrm{A} 73+\mathrm{A} 74-\mathrm{A} 75-\mathrm{A} 76+\mathrm{A} 77+\mathrm{A} 78 \\
& -\mathrm{A} 79-\mathrm{A} 80-\mathrm{A} 81+\mathrm{A} 82-\mathrm{A} 83-\mathrm{A} 84 \\
& \begin{array}{l}
\mathrm{A} 61=\frac{\mathrm{GrA} 37 \operatorname{Cos}[\alpha]}{\mathrm{m} 9^{2}-\mathrm{m} 9+\left(M^{2}+N^{2}+i \omega\right)} \\
\mathrm{A} 62=\frac{\mathrm{GrA} 38 \operatorname{Cos}[\alpha]+(\mathrm{m} 4 \mathrm{~A} 15)}{\mathrm{m} 4^{2}-\mathrm{m} 4+\left(M^{2}+N^{2}+i \omega\right)} \\
\mathrm{A} 63=\frac{\mathrm{GrA} 45 \operatorname{Cos}[\alpha]+(\mathrm{m} 3 \mathrm{~A} 22)}{\mathrm{m} 3^{2}-\mathrm{m} 3+\left(M^{2}+N^{2}+i \omega\right)} \\
\mathrm{A} 64=\frac{\mathrm{GrA} 46 \operatorname{Cos}[\alpha]+(\mathrm{m} 2 \mathrm{~A} 23)}{\mathrm{m} 2^{2}-\mathrm{m} 2+\left(M^{2}+N^{2}+i \omega\right)} \\
\mathrm{A} 66=\frac{\mathrm{GrA} 47 \operatorname{Cos}[\alpha]+(\mathrm{m} 1 \mathrm{~A} 24)}{\mathrm{m} 1^{2}-\mathrm{m} 1+\left(M^{2}+N^{2}+i \omega\right)} \\
\mathrm{A} 67=\frac{\mathrm{GrA} 57 \operatorname{Cos}[\alpha]}{\mathrm{m} 8^{2}-\mathrm{m} 8+\left(M^{2}+N^{2}+i \omega\right)} \\
\mathrm{A} 68=\frac{\mathrm{GrAs}[\alpha]}{\mathrm{m} 6^{2}-\mathrm{m} 6+\left(M^{2}+N^{2}+i \omega\right)} \\
\mathrm{A} 69=\frac{\mathrm{m} 5 \mathrm{~A} 14}{\mathrm{~m} 5^{2}-\mathrm{m} 5+\left(M^{2}+N^{2}+i \omega\right)}
\end{array}
\end{aligned}
$$




$$
\begin{aligned}
& \mathrm{A} 70=\frac{\mathrm{GrA39} \operatorname{Cos}[\alpha]+(2 \mathrm{~m} 3 \mathrm{~A} 16)}{\mathrm{m} 3^{2}-\mathrm{m} 3+\left(M^{2}+N^{2}+i \omega\right)} \\
& \mathrm{A} 71=\frac{\mathrm{GrA} 40 \operatorname{Cos}[\alpha]+(2 \mathrm{~m} 2 \mathrm{~A} 17)}{\mathrm{m} 2^{2}-\mathrm{m} 2+\left(M^{2}+N^{2}+i \omega\right)} \\
& \mathrm{A} 72=\frac{\mathrm{GrA} 41 \operatorname{Cos}[\alpha]+(2 \mathrm{~m} 1 \mathrm{~A} 18)}{\mathrm{m} 1^{2}-\mathrm{m} 1+\left(M^{2}+N^{2}+i \omega\right)} \\
& \mathrm{A} 73=\frac{\mathrm{GrA} 42 \operatorname{Cos}[\alpha]+(\mathrm{m} 2+\mathrm{m} 3) \mathrm{A} 19}{(\mathrm{~m} 2+\mathrm{m} 3)^{2}-(\mathrm{m} 2+\mathrm{m} 3)+\left(M^{2}+N^{2}+i \omega\right)} \\
& \mathrm{A} 74=\frac{\mathrm{GrA} 43 \operatorname{Cos}[\alpha]+(\mathrm{m} 1+\mathrm{m} 3) \mathrm{A} 20}{(\mathrm{~m} 1+\mathrm{m} 3)^{2}-(\mathrm{m} 1+\mathrm{m} 3)+\left(M^{2}+N^{2}+i \omega\right)} \\
& \mathrm{A} 75=\frac{\mathrm{GrA} 44 \operatorname{Cos}[\alpha]+(\mathrm{m} 1+\mathrm{m} 2) \mathrm{A} 21}{(\mathrm{~m} 1+\mathrm{m} 2)^{2}-(\mathrm{m} 1+\mathrm{m} 2)+\left(M^{2}+N^{2}+i \omega\right)} \\
& \mathrm{A} 76=\frac{\operatorname{GrA} 48 \operatorname{Cos}[\alpha]}{(\mathrm{m} 3+\mathrm{m} 8)^{2}-(\mathrm{m} 3+\mathrm{m} 8)+\left(M^{2}+N^{2}+i \omega\right)} \\
& \mathrm{A} 77=\frac{\mathrm{GrA} 49 \operatorname{Cos}[\alpha]}{(\mathrm{m} 3+\mathrm{m} 7)^{2}-(\mathrm{m} 3+\mathrm{m} 7)+\left(M^{2}+N^{2}+i \omega\right)} \\
& \mathrm{A} 78=\frac{\operatorname{GrA50\operatorname {Cos}}[\alpha]}{(\mathrm{m} 3+\mathrm{m} 6)^{2}-(\mathrm{m} 3+\mathrm{m} 6)+\left(M^{2}+N^{2}+i \omega\right)} \\
& \mathrm{A} 79=\frac{\operatorname{GrA51Cos}[\alpha]}{(\mathrm{m} 2+\mathrm{m} 8)^{2}-(\mathrm{m} 2+\mathrm{m} 8)+\left(M^{2}+N^{2}+i \omega\right)} \\
& \mathrm{A} 80=\frac{\operatorname{GrA} 52 \operatorname{Cos}[\alpha]}{(\mathrm{m} 2+\mathrm{m} 7)^{2}-(\mathrm{m} 2+\mathrm{m} 7)+\left(M^{2}+N^{2}+i \omega\right)} \\
& \mathrm{A} 81=\frac{\operatorname{GrA53Cos}[\alpha]}{(\mathrm{m} 2+\mathrm{m} 6)^{2}-(\mathrm{m} 2+\mathrm{m} 6)+\left(M^{2}+N^{2}+i \omega\right)} \\
& \mathrm{A} 82=\frac{\operatorname{GrA} 54 \operatorname{Cos}[\alpha]}{(\mathrm{m} 1+\mathrm{m} 8)^{2}-(\mathrm{m} 1+\mathrm{m} 8)+\left(M^{2}+N^{2}+i \omega\right)} \\
& \mathrm{A} 83=\frac{\operatorname{GrA55\operatorname {Cos}[\alpha ]}}{(\mathrm{m} 1+\mathrm{m} 7)^{2}-(\mathrm{m} 1+\mathrm{m} 7)+\left(M^{2}+N^{2}+i \omega\right)} \\
& \mathrm{A} 84=\frac{\operatorname{GrA56\operatorname {Cos}[\alpha ]}}{(\mathrm{m} 1+\mathrm{m} 6)^{2}-(\mathrm{m} 1+\mathrm{m} 6)+\left(M^{2}+N^{2}+i \omega\right)}
\end{aligned}
$$

\section{References}

[1] Amos, E. and Omamoke. E., (2018). MHD Free Convetive Flow over an Inclined Porous Surface with Variable Suction and Radiation Effects. International Journal of Applied Science and Mathematical Theory, ISSN 2489-009X, Vol. 4, No. 3.

[2] Israel-Cookey, C., Ogulu A. and V. B. Omubo-Pepple. (2002). Influence of viscous dissipation and radiation on unsteady MHD free-convection flow past an infinite heated vertical plate in a porous medium with time-dependent suction, International Journal of Heat and Mass Transfer 46 (2003) 2305-2311 Received 1 August 2002; received in revised form 9.

Theoretical and Applied Mathematics, 2, 3, pp 217-231.

[4] Venkateswarlu, M., (2015), Unsteady MHD free convective Heat and Mass Transfer in a Boundary Layer Flow Past a vertical Permeable plate with Thermal Radiation and Chemical Reaction. Internal Conference of Computational Heat and Mass Transfer, Procedia Engineering 127, 791 - 799.

[5] Sankar, Reddy, Sudheer, Babu, M., Narayana, P. V. and Umamaheswara, Reddy, D., (2011). Radiation and Chemical Reaction Effect on an Unsteady MHD Convection Flow Past a Vertical Moving Porous Plate Embedded in a Porous Medium with Viscous Dissipation, Advances in Applied Research, 2 (5):226-239, ISBN:0976-8610.

[6] Durga, Prasad, P., Kiran, Kumar, R. V. M. S. and Varma, S. V. K., (2016). Heat and Mass Transfer for the MHD Flow of Nano-Fluid with Radiation Absorption, Ain Shams Engineering Journal, www.sciencedirect.com. 
[7] Madhusudhana, Rao, B., Raju, M. C., Viswanath, Reddy, G. and Varma, S. V. K., (2013). Unsteady MHD Free Convective Double Diffusive and Dissipative Visco-Elastic Fluid Flow in Porous Medium with Suction, International Journal of Advances in Science and Technology, Volume 7, number 2, ISSN: 2229-5216.

[8] Venkateswarlu, M., Venkata, Lakshmi, D. and Naga, Malleswara, Rao, K., (2016). Soret, Hall Current, Rotation, Chemical Reaction and Thermal Radiation Effects on Unsteady MHD Heat and Mass Transfer Natural Convection Flow Past an Accelerated Vertical Plate. J. KSIAM, Volume 20, number 3, pp 203-224, DOI-10.12941.

[9] Harinath, Reddy, S., Raju, M. C. and Reddy, Keshava, E., (2016). Soret and Defour Effects on Radiation Absorption Fluid in the Presence of Exponentially Varying Temperature and Concentration in a Conducting Field, Special Topics and Reviews in Porous Media-An International Journal 7(2):115-129.

[10] Rojaa, T., Sankar, Reddy, B. and Bhaskar, Reddy, (2013). Radiation and Chemical Reaction Effect on MHD Free Convection Flow of a Micro-polar Fluid Bounded by Vertical Infinite Surface with Viscous Dissipation and Constant Suction, International Journal of Advanced Research, IJOAR.org, Volume 1, Issue 3, ISSN: 2320-9135.

[11] Venkateswarlu, M., Ramana, Reddy, G. V. and Lakshmi, D. V., (2013). Unsteady MHD Flow of a Viscous Fluid Past a Vertical Porous Plate under Oscillatory Suction Velocity, Advances in Applied Research, 4(6):52-67, ISSN: 0976-8610.

[12] Ojemeri, G., Onwabuya, I. O. and Abdusalam, S., Effect of Soret and Radial Magnetic Field of a Free Convective Slip Flow in a Viscous Reactive Fluid Towards a Vertical Porous Cylinder, Continental Journal of Applied Sciences, 14 (1): 2545 DOI:10.5281, ISSN: 1597-9928.
[13] Reddy, M. G. and Reddy, N. B., (2009). Radiation and Mass Transfer Effects on Unsteady MHD Free Convection Flow of an Incompressible Viscous Fluid Past a Moving Vertical Cylinder, Journal of Applied Mathematics and Mechanics, Volume 6, pp 96-110.

[14] Rammana, Reddy, J. V., Sugunamma, V., Sandeep, N. and Sulochana, C., (2016). Influence of Chemical Reaction, Radiation and Rotation on MHD Nano Fluid Flow Past a Permeable Flat Plate in Porous Medium, Journal of the Nigerian Mathematical Society, Volume 35, pp 48-65.

[15] Krupa, Lakshmi, K. L., Gireesha, B. J., Rama, Gorla, S. R. and Mahanthesh, B., (2016). Effects of Diffusion on Two Phase Boundary Layer Flow Past a Stretching Sheet with Fluid-Particles Suspension and Chemical Reaction: A numerical Study, Journal of the Nigerian Mathematical Society, Volume 35, pp 66-81.

[16] Salawu, S. O. and Dada, M. S., (2016). Radiative Heat Transfer of Variable Viscosity and Thermal Conductivity Effects on Inclined Magnetic Field with Dissipation in a nonDarcy Medium, Journal of the Nigerian Mathematical Society, Volume 35, pp 93-106.

[17] Das, K., (2011). Effect of Chemical Reaction and Thermal Radiation on Heat and Mass Transfer Flow of MHD Micropolar Fluid in a Rotary Frame of Reference. International Journal of Heat and Mass Transfer, 54:3505-13.

[18] Ekakitie, Omamoke and Emeka, Amos, (2020). The Impact of Chemical Reaction and Heat Source on MHD Free Convection Flow over an Inclined Porous Surface, Internal Journal of Scientific and Research Publication, Volume 10, Issue $5, \quad$ ISSN:2250-3153, 10.29322/IJSRP.10.05.2020.P10103. 